\title{
IL RIPENSAMENTO DEL WELFARE STATE IN GERMANIA E NEGLI STATI UNITI
}

\author{
di Jens Alber
}

Universalismo e selettività nel dibattito sul welfare state

L'età dell'oro del welfare state sembra essersi conclusa. I cambiamenti demografici e l'intensificarsi della competizione internazionale in un'epoca di globalizzazione hanno messo a dura prova i sistemi di welfare. Sebbene non vi siano indicazioni di un rifiuto generale dello stato sociale (welfare backlash) tra l'opinione pubblica occidentale ${ }^{1}$, gli elettori spesso scelgono partiti neo-conservatori che promettono di tagliare i programmi sociali e ridurre le tasse. In questa nuova fase di stagnazione e ridimensionamento dello stato sociale, la questione di quali fattori determinino la vulnerabilità di particolari programmi o espongano alcuni sistemi di welfare state a una politica di tagli ha assunto un ruolo centrale nel dibattito accademico.

Autori come Esping-Andersen (1990), Flora (1986) o Le Grand e Winter (1987) sono d'accordo nel ritenere che l'espansione e la capacità di tenuta degli schemi di welfare variano a seconda del loro grado di universalismo. Questa affermazione è tradizionalmente legata a due ipotesi. In base alla prima, il grado in cui il processo di redistribuzione pubblica attraverso il bilancio riflette gli interessi di gruppi particolari dipende in larga parte dalle risorse di potere dei gruppi stessi. Quei gruppi che non sono in grado di mettere sul piatto in modo credibile la minaccia del ritiro di beni e servizi di vitale importanza per il funzionamento dell'intera società, che possiedono scarse capaci-

Una prima versione di questo lavoro è stata presentata al panel «Inequality and Universalism» del $92^{\circ}$ Meeting annuale dell'American Political Science Association (San Francisco, 31 agosto 1996).

1 Cfr. Borre e Scarborough (1995); Svallfors (1993); Roller (1992); Shapiro e Young (1989); Taylor-Gooby (1985a, 1985b); Coughlin (1980).

RIVISTA ITALIANA DI SCIENZA POLITICA / a. XXVII, n. 1, aprile 1997 
tà organizzative, o che non dispongono di un numero rilevante di voti tenderanno ad essere trascurati nel processo di redistribuzione politica (Offe 1972; Geißler 1976; Korpi 1978). Dal momento che le classi medio-alte dispongono in maniera maggiore di queste risorse di potere, i programmi sociali si diffondono e si consolidano nella misura in cui questi gruppi di interesse sono inclusi.

La seconda ipotesi si ricollega al tema della formazione delle coalizioni politiche e al fatto che in presenza di una crescente pressione fiscale la predisposizione al rifiuto del welfare state tende ad aumentare (Wilensky 1975; Esping-Andersen 1990). Là dove esistono dei programmi selettivi che costringono le classi medie a ricoprire un unico ruolo, vale a dire quello di finanziare il costo dei programmi sociali, la probabilità di un rifiuto del welfare cresce insieme alla pressione fiscale. Là dove, invece, schemi universali di welfare estendono la copertura alle classi medie e forniscono prestazioni generose non solo per i poveri, le classi medie si percepiscono, oltre che come meri contribuenti, anche come beneficiari di tali prestazioni e, di conseguenza, sviluppano un interesse alla difesa dei programmi stessi. Esping-Andersen $(1990,33)$ sintetizza questa tesi affermando che i modelli di stato sociale che tutelano la classe media forgiano la lealtà della stessa classe media.

L'ipotesi che collega universalismo e classi medie presenta numerose implicazioni interessanti e verificabili. Suggerisce, in primo luogo, che il grado di sostegno pubblico per i diversi programmi sociali varia a seconda del grado di inclusione della classe media o universalismo. Sebbene le inchieste d'opinione forniscano ancora prove scarse, fino ad ora $\mathrm{i}$ dati disponibili sembrano concordare con questa ipotesi ${ }^{2}$. Taylor-Gooby riassume così tali conclusioni: «i pregiudizi maggiori sembrano rivolgersi contro le prestazioni selettive» $(1985 \mathrm{~b}, 78)$.

Una seconda ipotesi verificabile è la seguente: i programmi sociali universali che includono le classi medie si diffondono in modo più veloce dei programmi selettivi relativi ai soli gruppi meno abbienti, in genere male organizzati e con scarse capacità di mobilitazione. Questo significa che durante la fase di espansione del welfare state si è andato ampliando il divario tra le prestazioni per i settori della società meglio organizzati e le prestazioni per i gruppi marginali, come ad esempio gli anziani più

2 Si vedano i riferimenti bibliografici della nota 1 . 
deboli o i malati mentali, privi di consistenti risorse di potere. Fino ad ora sono state fatte poche ricerche sistematiche su questo tema e le poche prove disponibili sembrano troppo eterogenee per poter suffragare in modo convincente questa ipotesi nella sua formulazione generale. Per giungere a qualche conclusione si dovrebbero prendere in considerazione le variazioni che interessano sia i singoli programmi che i singoli paesi. Infatti, mentre i programmi per gli anziani deboli si sono sviluppati meglio di quelli per i malati mentali, alcuni paesi europei, come la Danimarca e l'Olanda, hanno fatto molto di più nel campo dei servizi assistenziali alle persone in difficoltà di altri, come la Germania $^{3}$. Il risultato più significativo è che persino in paesi ritardatari come la Germania le prestazioni pro capite per le persone bisognose si sono diffuse ad un tasso uguale o persino maggiore dei benefici pro capite per i gruppi più centrali all'interno della forza lavoro ${ }^{4}$. Questi dati contrastano con l'idea che i programmi per i gruppi marginali con scarse risorse di potere siano stati trascurati nella fase di espansione del welfare state.

Da ultimo, l'ipotesi della classe media, così come è stata sviluppata dai sociologi, ha una ulteriore implicazione di immediato interesse per la recente fase di ridimensionamento del welfare state. Mentre gli economisti tendono a sottolineare l'efficacia e l'efficienza dei programmi di protezione sociale mirati (targeted), l'approccio sociologico suggerisce che gli schemi selettivi che escludono le classi medie producono un effetto a spirale verso il basso che determina il continuo abbassamento delle prestazioni. Dal momento che questi schemi riducono la maggioranza della popolazione al ruolo di contribuente, $\mathrm{i}$ beneficiari rappresentano una piccola minoranza che, rimasta priva di partner coalizionali, è troppo debole per resistere agli attacchi congiunti delle coalizioni di contribuenti e dei detentori di cariche pubbliche che in un'epoca di austerità cercano di contenere il deficit pubblico.

$3 \mathrm{Si}$ fa riferimento qui ad alcuni risultati del tutto preliminari emersi da due progetti di ricerca in cui sono stato coinvolto. Si è trattato, in primo luogo, di un convegno sull'offerta di servizi sanitari tenutosi al Max-Planck-Institut für Gesellschaftsforschung a Colonia nel 1990, i cui risultati sono stati pubblicati in una serie di articoli apparsi nel numero speciale Comparative Health Policy sulla rivista «Journal of Health Politics, Policy and Law», vol. 17, n. 4, 1992. In secondo luogo, si fa riferimento all'European Commission's Observatory on Social and Economic Policies for Older People in Europe, i cui risultati sono stati pubblicati in Walker, Alber e Guillemard (1993) e Alber (1995).

4 Sugli sviluppi tedeschi cfr. Alber (1992) e Schölkopf (1996). 
L'ipotesi che collega universalismo e classi medie implica pertanto che i programmi selettivi debbano diventare il primo obiettivo di una politica di ridimensionamento. Molti autori prevedono infatti processi di crescente segmentazione e marginalizzazione nei welfare state occidentali insieme all'emergere di nuove forme di povertà e persino di una nuova sottoclasse che sarà sempre più emarginata dal progresso delle condizioni di vita dei settori centrali della società (si vedano Tennstedt e Leibfried 1985; Dahrendorf 1988).

Dall'altro lato, le ricerche sopra menzionate sullo sviluppo dei servizi sociali per i gruppi con scarso potere organizzativo suggeriscono che lo sviluppo della politica sociale non può seguire rigidamente una logica di political economy. Pertanto le decisioni di policy riguardanti i gruppi marginali potrebbero essere determinate non solo in base all'analisi politica dei costi/ benefici $o$ in base alla distribuzione delle risorse di potere tra gruppi specifici, ma anche in base a regole generali che definiscano che cosa costituisce uno standard minimo socialmente accettabile. Mentre in alcuni paesi tali regole potrebbero avere lo status di semplici convenzioni sociali, in altri potrebbero essere istituzionalizzate all'interno di un ordinamento legales. Nella misura in cui questo si verifica, non ci si può aspettare che i risultati di policy riflettano con immediatezza relazioni di potere.

Sfortunatamente, studi sistematici sui processi di ridimensionamento del welfare in una prospettiva comparata sono ancora del tutto assenti. Tuttavia, il lavoro pionieristico di Pierson (1994) sfida la dottrina corrente e definisce semplicistica l'idea che il grado di inclusione della classe media sia il fattore chiave che salvaguarda i programmi sociali dalle pressioni restrittive. Pierson propone tre ipotesi: 1) i programmi selettivi, piuttosto che quelli universali, sono usciti vincitori dalla fase di ridimensionamento del welfare in Gran Bretagna e in America ${ }^{6}$; 2) la vulnerabilità dei programmi sociali dipende da fattori più complessi che non il loro semplice impianto selettivo o universale (Pierson 1994, 6 e 3) i tagli sono il risultato di un processo poli-

\footnotetext{
5 In Germania, per esempio, la costituzione definisce lo Stato tedesco come uno «stato sociale». Da questa definizione conseguono certe responsabilità sociali inalienabili.

$6 \ll$ Se negli anni ottanta i programmi sociali universali sono stati i grandi sconfitti programmatici, $i$ più grandi vincitori sono stati in realtà $i$ programmi mirati» (Pierson 1994, 103).
} 
tico che segue una specifica logica politica che varia a seconda dei feedback di policy radicati nella struttura di ogni singolo programma e a seconda dei costi politici che si accompagnano alle riforme.

Dal momento che la confutazione di Pierson dell'ipotesi che collega universalismo e classi medie è empiricamente dimostrata e ampiamente citata, intendo innanzitutto analizzare fino a che punto si può ritenere che il contributo di Pierson abbia realmente scosso la dottrina corrente. In seguito, intendo esaminare se i programmi selettivi hanno avuto lo stesso destino in Germania e negli Stati Uniti e fino a che punto sviluppi differenti possano essere attribuiti alle strutture dei diversi programmi sociali.

I programmi sociali mirati sono davvero usciti vincenti dal ridimensionamento del welfare? Un riesame delle ipotesi di Pierson

Pierson collega l'ipotesi che i programmi selettivi siano usciti vincenti dalla fase di ridimensionamento del welfare all'idea che nelle democrazie occidentali «gli interventi di ridimensionamento sono in genere impopolari» $(1994,33)$. Se, come dimostrano i sondaggi d'opinione, non c'è stato un rifiuto del welfare state e i programmi sociali continuano a godere del sostegno popolare, questo rende le politiche di ridimensionamento un esercizio volto ad «evitare il biasimo» (blame avoidance) piuttosto che a «vantare il credito» (credit-claiming) $(1994,18)^{7}$. Sulla base di questa premessa, Pierson ritiene che gli interventi restrittivi possano avere luogo solo alle seguenti condizioni (1994, 4-9; 1996, 156):

- $i$ governi sono in grado di ridurre la visibilità dei loro tagli (un'interessante implicazione di questa ipotesi è che i programmi in cui le responsabilità sono centralizzate a livello nazionale tendono ad essere meno predisposti ai tagli dei programmi decentrati le cui responsabilità sono diffuse); Pierson (1994, 169) definisce questa tendenza come la «caratteristica

7 Per quanto questo concetto sia diventato popolare nel corso degli anni ottanta, il motto «concentrare la gloria, diffondere il biasimo», era già noto negli anni settanta e può essere considerato l'immagine speculare della famosa frase di John F. Kennedy secondo la quale «il successo ha molte paternità mentre il fallimento è orfano» (cfr. anche Klein 1981, 177). 
del federalismo che facilita gli interventi restrittivi» (si veda anche Pierson 1995);

- i gruppi di interesse che sostengono un programma specifico sono deboli (una condizione che, seguendo la logica neoistituzionalista, dipende principalmente dalla struttura del programma, la quale può impedire o facilitare la formazione di gruppi di interesse) oppure il governo trova il modo di impedire la loro mobilitazione;

- i governi sono in grado di compensare coloro che risulteranno penalizzati dalla politica dei tagli e di escogitare nei confronti dell'elettorato strategie di divide et impera;

- la struttura dei programmi produce dei feedback di policy che sono favorevoli alla riforma. L'idea qui è che un sistema pensionistico maturo sia più difficile da riformare - usando la terminologia di Pierson dovremmo dire sia più «imprigionato» (locked-in) - di uno schema introdotto solo recentemente e che copre un numero limitato di beneficiari, e che sia difficile che le soglie di reddito previste per l'accesso ad alcune prestazioni scendano al di sotto di un certo livello;

- condizioni di grave crisi finanziaria conferiscono credibilità a coloro che sostengono che vi sia un bisogno funzionale di politiche restrittive.

Siccome le prestazioni selettive dei gruppi sociali con bassi redditi sono in genere assai poco generose, nel caso di questi schemi vi sono poche opportunità di minimizzare $i$ costi visibili che derivano dall'introduzione di tagli (Pierson 1994, 170). Essendo $i$ tagli un costo visibile, è probabile che $\mathrm{i}$ governi che decidono di riformare tali schemi debbano fare i conti con la questione dell'«equità» $(1994,127)$. Dal momento che i politici mirano ad essere rieletti, essi sono disposti a cedere su questo fronte e a programmare cambiamenti di minor portata tralasciando i più ampi progetti di ridimensionamento dei programmi selettivi.

Pierson insiste nell'analizzare il successo dei singoli programmi o i tentativi di ridimensionamento alla luce degli specifici feedback di policy e delle opportunità di policy. Questo approccio è certamente stimolante ${ }^{8}$. Tuttavia, il concetto di blame avoidance implica una interpretazione chiaramente unidimensio-

8 Per studi precedenti sullo sviluppo del welfare state che adottano lo stesso approccio cfr. Heclo (1974), Orloff e Skocpol (1984), Orloff (1993), insieme alle varie analisi dei processi di ridimensionamento in Goodin e Le Grand (1987) e Morris (1988). 
nale del processo politico nelle democrazie occidentali. I risultati di politica pubblica sono descritti come una funzione unidimensionale della relazione tra politici ed elettori: i primi mirano ad essere rieletti e per questo hanno bisogno del sostegno della maggioranza degli elettori e sono pronti a modificare la loro agenda di policy sulla base degli interessi dell'opione pubblica. A differenza di Hirschman (1970), che ha indicato le dimensioni dell'exit e della voice come meccanismi di influenza, e dei neo-marxisti come O'Connor (1973) o Offe (1984), che hanno evidenziato la tensione tra le funzioni di legittimazione e accumulazione, Pierson considera solo una dimensione della politics democratica, vale a dire il punto di vista dei detentori di cariche pubbliche che reagiscono agli elettori o ne prevengono la reazione $(1994,17)^{9}$.

L'attenzione esclusiva sulla politica elettorale (si veda anche Pierson 1996, 149) lascia irrisolte alcune importanti questioni. Se evitare il biasimo e ottenere il sostegno pubblico rappresentano le due determinanti cruciali dello sviluppo delle politiche, è difficile capire perché i programmi selettivi, che stando ai risultati delle ricerche comparate riscuotono un sostegno decisamente inferiore a quello ottenuto dai programmi universali, dovrebbero risultare vincitori nel processo di ridimensionamento. Se gli elettori costituiscono un freno cruciale per le politiche pubbliche e gli interventi di ridimensionamento del welfare sono in genere ritenuti impopolari, ci si dovrebbe innanzitutto chiedere per quale ragione i partiti neo-conservatori hanno vinto le elezioni. La maggioranza degli elettori non li ha votati (tra le altre ragioni) proprio sulla base della loro agenda di riforma del welfare state resa nota durante la campagna elettorale? Se è così, e se è vero che i partiti devono stare attenti a non perdere il sostegno del loro nucleo duro di elettori, evitare il biasimo per i governi conservatori avrebbe dovuto implicare innanzitutto e soprattutto l'attuazione di una politica di tagli così come era stata preannunciata agli elettori. L'elettorato conservatore non era tanto preoccupato da questioni di «equità» quanto piuttosto dagli effetti perversi del welfare sugli incentivi al lavoro e sulla competitività delle imprese. La sua aspirazione era quella di ripristinare una certa distanza tra il livello delle prestazioni godu-

9 Per una discussione di taglio empirico riguardante la misura in cui l'opinione pubblica esercita una influenza sul processo di policy-making sociale cfr. Shapiro e Young $(1989,85-87)$. 
te senza lavorare e il livello dei redditi da lavoro (una sorta di ritorno al principio della less eligibility: chi è abile al lavoro deve essere incoraggiato a lavorare e non a vivere di sussidi).

Come sottolinea Pierson, la propensione dei governi conservatori a produrre i risultati di policy che i loro gruppi di riferimento si aspettano sarà controbilanciata da una importante tendenza: i costi dei tagli sono altamente concentrati e pertanto marcatamente e penosamente visibili ai loro destinatari, mentre $\mathrm{i}$ benefici che ne deriveranno alla platea di coloro che sono favorevoli ai tagli sono generalmente dispersi e molto meno visibili (Pierson 1994, 13; 1996, 145). Se sia davvero questo ciò che si verifica è prima di tutto una questione empirica, e qualsiasi tendenza di questo tipo sarà controbilanciata se i gruppi che beneficiano dei trasferimenti e dei servizi sociali mostrano un tasso di partecipazione elettorale considerevolmente più basso delle classi medie che finanziano il welfare state con i loro contributi. A quanto mi consta, questo è di solito il caso delle democrazie occidentali, e soprattutto degli Stati Uniti, dove le prestazioni sono concentrate tra le minoranze etniche che raramente votano ${ }^{10}$.

Bisogna anche ricordare che i voti non sono il solo meccanismo di influenza politica e la sola risorsa di potere di un gruppo di interesse. In un'epoca di globalizzazione e di mercati finanziari internazionali le minacce di defezione di coloro che detengono i capitali hanno guadagnato un peso politico notevo$\mathrm{le}^{11}$. Anche se questa influenza potrebbe esercitare, attraverso il controllo delle risorse vitali, solo un effetto indiretto sui risultati elettorali - così come suggerisce Pierson $(1996,149)$-, la minaccia di non concedere a un partito un sostegno finanziario per la sua campagna elettorale è invece più immediata e avrà un impatto diretto sulle dinamiche elettorali.

Riassumendo, bisogna concettualizzare i governi come entità sottoposte a pressioni politiche multi-dimensionali. I detentori di cariche pubbliche non subiscono pressioni solo da coloro che ricevono trasferimenti e servizi; devono anche prendere in considerazione gli interessi pro-tagli di coloro che finanziano $\mathrm{i}$

10 Per i modelli di voto cfr. Piven e Cloward (1988, 162); per la fruizione di prestazioni sociali si veda U.S. House of Representatives Committee on Ways and Means $(1993,1994)$.

11 Sebbene Pierson sia occasionalmente consapevole delle pressioni economiche (1994, 180; 1996, 148-149), egli non prende in considerazione questo fattore in modo sistematico. 
programmi sociali e che sono sottoposti ad un crescente peso fiscale. Inoltre, i governi devono essere considerati degli attori indipendenti che perseguono obiettivi multi-dimensionali. Da un lato mirano ad essere rieletti; dall'altro propugnano obiettivi sostantivi di politica pubblica sulla base di idee specifiche di ciò che costituisce un ordine sociale giusto; inoltre, attori statali indipendenti perseguono anche interessi fiscali particolari (come, ad esempio, scaricare i costi delle politiche pubbliche su altri attori o manipolare il deficit) che tagliano in modo trasversale le loro idee programmatiche mentre gravano in larga parte sulle strutture finanziarie di programmi sociali specifici.

Prima di fare congetture sull'impatto dei diversi fattori esplicativi degli interventi restrittivi, è necessario chiarire che cosa esattamente deve essere spiegato. Questo implica misurare, attraverso degli indicatori riproducibili, il grado in cui alcuni singoli programmi sociali hanno subito degli interventi restrittivi. Ed è proprio da questo punto di vista che lo studio di Pierson, che pure ha fatto scuola, sembra mostrare le maggiori debolezze.

Le principali conclusioni descrittive di Pierson possono essere riassunte in tre punti:

- c'è stato «un modello dominante di continuità nell'ambito delle politiche sociali» $(1994,179)$;

- i programmi universali hanno perso mentre quelli mirati sono usciti vincenti dalla fase di ridimensionamento del welfare (1994, 103, 127-128, 170);

- gli interventi restrittivi programmatici - cioè i tagli a singoli programmi - «in Gran Bretagna in genere si sono spinti più avanti rispetto agli Stati Uniti» $(1994,131)$, mentre «l'amministrazione Reagan è quella che ha avuto più successo» per quanto riguarda gli interventi restrittivi sistematici - cioè gli sforzi per erodere dalle fondamenta la politica sociale, decurtando $\mathrm{i}$ finanziamenti al welfare state o indebolendo le basi del suo sostegno popolare $(1994,162)$.

Il problema è che nessuna di queste conclusioni ${ }^{12}$ è riproducibile in modo tale che il lettore possa giungere agli stessi risultati. Infatti, Pierson non ha fatto nessun tentativo di operazionalizzare o di misurare il grado di continuità dei programmi di welfare state. Come nota lo stesso Pierson, «è probabile che let-

12 In realtà ci stiamo riferendo più all'interpretazione delle conclusioni ottenute dalle fonti primarie che non alle conclusioni stesse. 
tori diversi diano risposte differenti» alla domanda su «cosa costituisce un cambiamento radicale» $(1994,145)$. Il suo tentativo di stabilire in modo sistematico se i tagli programmatici ai singoli programmi siano stati maggiori in Gran Bretagna o negli Stati Uniti si ferma alla compilazione di tabelle costruite in base a una classificazione piuttosto grezza (impatto forte, moderato e debole: 1994, 143). Inoltre, molto spesso il livello di precisione delle descrizioni nel testo è questo: «sebbene sia difficile sostenere che i cambiamenti negli Stati Uniti siano stati drammatici, il quadro inglese è meno chiaro» $(1994,145)$. Che classificazioni così imprecise non siano una base empirica sufficientemente solida per proporre interpretazioni teoriche sul successo degli interventi restrittivi su singoli programmi diventa chiaro se si confrontano i risultati di Pierson con quelli delle fonti primarie da lui stesso usate per ottenere i suoi dati.

In una analisi estremamente accurata, Atkinson e Micklewright hanno descritto lo sviluppo dell'assicurazione contro la disoccupazione in Gran Bretagna. Dopo aver elencato la riduzione del livello delle prestazioni con l'abolizione dell'integrazione collegata al reddito, l'abolizione dell'integrazione per i figli a carico, la tassazione delle prestazioni, il quadruplice aumento del periodo massimo di carenza delle prestazioni, e diverse altre misure, gli autori sintetizzano le loro conclusioni nel modo seguente: «dal 1979 si è assistito ad uno spostamento consistente dalle prestazioni di tipo assicurativo verso forme selettive di assistenza per i disoccupati. Senza un dibattito pubblico, c'è stata una alterazione dei principi sottostanti la protezione economica per $\mathrm{i}$ disoccupati» (Atkinson e Micklewright $1989,145)$. Per quanto Pierson $(1994,107)^{13}$ citi queste conclusioni in modo accurato nel suo libro, tuttavia definisce «debole» l'impatto degli interventi restrittivi adottati in Gran Bretagna per quanto riguarda gli schemi di sostegno al reddito $(1994,143)^{14}$. Nel suo articolo più recente Pierson così descrive le trasformazioni che hanno avuto luogo in Gran Bretagna: «mentre il governo metteva a segno alcuni significativi tagli incrementali nei vari programmi sociali, altri sforzi radicali in sen-

13 Pierson fa riferimento all'articolo pubblicato in Dilnot e Walker (1989) mentre qui si fa riferimento all'articolo pubblicato in Atkinson (1989).

14 D'altronde, in altre parti del libro Pierson parla di interventi restrittivi in materia di disoccupazione «estensivi» e «consistenti» e di «morte per dissanguamento a causa di migliaia di tagli» (Pierson 1994, 5, 101, 107). 
so restrittivo fallirono, spesso con costi politici elevati» (1996, 163). La questione a questo punto è quella di capire che cosa costituisca «uno sforzo radicale in senso restrittivo».

Una discrepanza ancora più marcata si può riscontrare nella classificazione ed interpretazione dello sviluppo americano. Bawden e Palmer, in un'accurata analisi dei cambiamenti di bilancio durante l'amministrazione Reagan, illustrano come le spese per l'Aid for Families with Dependent Children (Afdc), rispetto al periodo precedente alla presidenza Reagan, siano state ridotte del $14 \%$ in base alle previsioni di spesa per il 1985 ; come un ammontare compreso tra l' $11 \%$ e il $14 \%$ dei beneficiari abbia perso il diritto a tali prestazioni; e come un altro $8 \%$ abbia visto le proprie prestazioni decurtate per un ammontare compreso tra 150 e 200 dollari al mese (Bawden e Palmer 1984, 192 e 364). Gli stessi autori documentano anche di tagli pari al $14 \%$ del bilancio per i buoni viveri (food stamps) da realizzarsi entro l'anno fiscale 1985 (rispetto alle previsioni di spesa antecedenti all'amministrazione Reagan); riferiscono che più di un milione sui 21 milioni aventi diritto sono stati eliminati dallo schema e che il livello reale delle prestazioni è stato ridotto per quasi tutti i beneficiari (1984, 367-368). Bawden e Palmer affermano anche che «i beneficiari degli schemi Afdc e Ssi (Supplemental Security Income) hanno comunque ottenuto un trattamento migliore durante l'amministrazione Reagan che non negli anni settanta» $(1984,193)$ e che «rispetto ai suoi obiettivi il presidente Reagan ha avuto meno successo nel tagliare quei programmi che fornivano prestazioni in denaro agli individui a basso reddito» $(1984,187 \text {, corsivo aggiunto })^{15}$; sintetizzano tuttavia le loro conclusioni affermando che «fino ad ora sotto l'amministrazione Reagan i poveri hanno continuato a diventare più poveri» $(1984,215)^{16}$.

15 Quando si misura il successo degli interventi restrittivi nei termini dei cambiamenti proposti dal presidente - conclusisi con meno di un terzo delle riduzioni programmate per i gruppi a basso reddito rispetto ad una riduzione di più della metà nell'assicurazione sociale e di quasi tre quarti nei programmi discrezionali di erogazione dei sussidi (Bawden e Palmer 1984, 187) - si devono ricordare le diverse dimensioni degli obiettivi retoricamente proclamati sui tagli. Più basso è stato l'obiettivo dei tagli programmato e più alta la probabilità che tagli contenuti si traducessero in alte percentuali di attuazione. Se i cambiamenti attuati vengono confrontati con il bilancio precedente invece che con i cambiamenti previsti, la sicurezza sociale risulta aver subito tagli pari al 5\%, di contro al $14 \%$ rispettivamente per l'Afdc e i buoni viveri (cfr. tab. 6.1 in Bawden e Palmer 1984, 185, in cui sono riportati $\mathrm{i}$ dati riguardanti $\mathrm{i}$ programmi specifici).

16 Riflettendo sulla distribuzione dei tagli ai singoli programmi, gli autori implici- 
Facendo riferimento ai dati di Bawden e Palmer, Edsall e Edsall $(1992,161)$ sintetizzano i tagli sotto la presidenza repubblicana in questo modo: «è chiaro che con una politica dei tagli mirata a programmi quali l'Afdc e i buoni viveri, l'amministrazione Reagan è riuscita a far gravare il peso delle riduzioni di spesa per lo più sulle minoranze»; «durante la presidenza Reagan la percentuale più ampia di tagli nella spesa sociale federale è stata attuata su programmi means-tested, indirizzati in larga parte alla popolazione di colore e amministrati, in molti casi, da un personale anch'esso di colore» $(1992,191-192)^{17}$.

Questi dati sui risultati dell'amministrazione Reagan sono notevolmente in contrasto con i risultati di Pierson. Infatti, egli definisce i programmi mirati come i vincitori del processo di ridimensionamento, richiama l'attenzione «sul successo limitato degli sforzi per tagliare i programmi per i poveri non invalidi», e descrive le prestazioni universali «come in qualche misura quelle più soggette ai cambiamenti» (Pierson 1994,128$)^{18}$. Sempre citando Pierson, i tagli più significativi ai programmi di sostegno al reddito si sono verificati «solo nel caso dei programmi in cui gli Stati e il governo federale avevano responsabilità in comune, vale a dire l'Afdc e l'assicurazione di disoccupazione» $(1994,115)^{19}$.

Riassumendo, sebbene autori diversi si riferiscano agli stessi dati, le interpretazioni sostantive che ne danno variano enormemente. Una ragione di queste discrepanze è che gli autori par-

tamente sviluppano una spiegazione funzionale nei termini dell'apprendimento politico. Dopo aver evidenziato che «il Congresso e le corti hanno esercitato una considerevole forza moderatrice delle intenzioni del presidente» propongono la seguente logica del processo di ridimensionamento: «il risultato è stato che i programmi ritenuti maggiormente inefficaci hanno subìto profondi tagli o sono stati eliminati mentre i programmi generalmente considerati efficaci non sono stati toccati o hanno subito dei cambiamenti alquanto modesti» (Bawden e Palmer 1984, 214).

17 Edsall e Edsall $(1992,192)$ documentano tagli di bilancio pari al $17,4 \%$ per l'Afdc e al $14,3 \%$ per i buoni viveri e così facendo citano male la loro fonte in base alla quale i tagli sarebbero stati pari al $14,3 \%$ per l'Afdc, al $13,8 \%$ per i buoni viveri e al $17,4 \%$ per l'assicurazione di disoccupazione (Bawden e Palmer 1984, 185; Edsall e Edsall citano la fonte come Palmer e Sawhill 1984, dando credito ai curatori del volume).

18 Anche se Pierson cita quasi tutte le conclusioni principali di Bawden e Palmer, egli continua ad affermare che «i buoni viveri hanno funzionato abbastanza bene» e cerca di sottostimare il significato dei tagli attuati aggiungendo che «tuttavia, molti dei tagli ai buoni viveri miravano solo a produrre dei buoni conti di bilancio e a minimizzare le trasformazioni reali» (Pierson 1994, 118).

19 Nella percezione di Pierson, naturalmente, questo è collegato alla struttura a più livelli che contribuisce a diffondere la responsabilità politica e ad evitare il biasimo individuale. 
tono da quesiti di ricerca diversi che dovrebbero essere attentamente tenuti separati. Domandarsi fino a che punto i governi conservatori hanno avuto successo nell'attuare gli obiettivi di politica da loro programmati è una cosa. Altra cosa è chiedersi fino a che punto i vari gruppi di beneficiari del welfare state hanno subìto dei tagli nelle loro prestazioni. Come sarà discusso in seguito più in dettaglio, rispondere a una di queste due domande non significa automaticamente fornire una risposta anche all'altra; è necessario, nelle nostre interpretazioni, stare molto attenti a non saltare da un livello di analisi all'altro. Osservazioni teoriche forti riguardanti la dinamica del processo di ridimensionamento dei singoli programmi sociali richiedono innanzitutto una più precisa misurazione delle variabili dipendenti che descrivono i tagli. Qualsiasi tentativo di proporre queste misurazioni deve partire da alcune regole di base sullo studio delle politiche di ridimensionamento del welfare e, da questo punto di vista, la ricerca pionieristica di Pierson rappresenta un utile punto di partenza.

Alcune regole di base per lo studio delle politiche di ridimensionamento del welfare

Pierson ha proposto tre regole di base per lo studio delle politiche dei tagli, che possono essere sintetizzate nel modo seguente $(1994,14-17)$ :

a) per comprendere a pieno l'effetto di una serie di cambiamenti più o meno grandi che hanno avuto luogo gradualmente nel corso del tempo (ad esempio, i cambiamenti nelle regole di indicizzazione) bisogna prendere in considerazione tagli di spesa sia di breve che di lungo periodo;

b) l'attenzione deve essere rivolta non solo alle spese e alla dimensione dei programmi, ma anche alla struttura degli schemi (così che spostamenti nell'erogazione di prestazioni sociali in una direzione maggiormente residualistica possano essere letti come interventi restrittivi anche se il livello delle spese rimane immutato);

c) occorre distinguere tra interventi restrittivi programmatici ed interventi restrittivi sistematici, che modificano la political economy delle politiche sociali future (o tagliando i finanziamenti al welfare state o indebolendo i gruppi di interesse che lo sostengono). 
Queste regole di base - soprattutto la prima - forniscono delle utili pietre miliari per qualsiasi studio delle politiche di ridimensionamento. Per garantire la replicabilità dei risultati raggiunti ed evitare interpretazioni confuse, queste regole devono essere tuttavia integrate da alcune indicazioni più specifiche, in grado di trasformare un approccio generale in operazionalizzazioni specifiche. Elenco qui di seguito cinque regole di base aggiuntive:

1) Occorre distinguere con chiarezza tra i cambiamenti di spesa aggregata a livello macro e i cambiamenti dei livelli di generosità delle prestazioni indotti istituzionalmente a livello micro.

I dati di bilancio sulla spesa non devono essere confusi con i dati sui livelli di generosità delle prestazioni. Sfortunatamente, la maggior parte degli studi empirici sugli interventi restrittivi si basa principalmente su dati di bilancio e su variazioni di spesa aggregata (Bawden e Palmer 1984), mentre in alcuni casi risulta poco chiaro quale sia il livello di analisi a cui si riferisce l'autore (Borchert 1995, 232; Pierson 1994, 119). Se la spesa aggregata di un certo programma - ad esempio quello contro la disoccupazione - cambia, non è chiaro se questo sia dovuto alle trasformazioni del contesto (il livello e la struttura della disoccupazione) o a cambiamenti nelle disposizioni legislative ${ }^{20}$. Per questa ragione uno studio della politica di ridimensionamento del welfare dovrebbe basarsi su dati istituzionali riguardanti i cambiamenti legislativi piuttosto che su dati di spesa aggregata ricavati dai bilanci.

2) Se l'attenzione è rivolta alle trasformazioni dei livelli di generosità delle prestazioni a livello micro, bisogna specificare quale delle seguenti operazionalizzazioni si presta meglio a misurare l'efficacia degli interventi restrittivi e a determinare chi vince e chi perde nel processo di ridimensionamento:

- la differenza tra i piani di intervento/riforma annunciati dal governo e quanto viene realmente attuato (questo indicatore può servire per misurare lo svuotamento di una legge di riforma

20 Indicatori più accurati, basati su dati aggregati quali il livello medio delle prestazioni (ottenuto dividendo la spesa aggregata per il numero di beneficiari), non possono spiegare adeguatamente i tagli. In un programma di assicurazione contro la disoccupazione che collega le prestazioni al reddito o ai contributi versati, l'ammontare medio delle prestazioni diminuirà pertanto anche in presenza di disposizioni legislative costanti se una percentuale crescente di disoccupati proviene da strati sociali con redditi bassi. Questo potrebbe, tuttavia, non essere considerato un indicatore dei tagli. 
in seguito all'azione di veto esercitata dai gruppi di pressione); oppure

- la differenza tra i criteri di eleggibilità prima e dopo l'introduzione di tagli (questo indicatore può cogliere quanto sono pesanti i tagli per quei gruppi che ne sono oggetto diretto).

Chiedersi fino a che punto $i$ governi riescano ad attuare $i$ loro obiettivi dichiarati non è la stessa cosa che chiedersi quali siano $\mathrm{i}$ gruppi di interesse che hanno più successo nel preservare costante l'ammontare delle prestazioni di cui sono beneficiari. Le due dimensioni possono variare indipendentemente, al punto che $\mathrm{i}$ due indicatori arrivano a produrre conclusioni sostantive ampiamente discordanti tra di loro. Così, per esempio, un governo può annunciare di tagliare metà della prestazione $\mathrm{A}$ e un terzo della prestazione B. Se riuscisse ad attuare almeno il $50 \%$ delle sue intenzioni, il grado di successo dell'intervento restrittivo sarebbe identico in base al primo indicatore; il secondo indicatore, invece, dimostrerebbe che il primo gruppo oggetto dell'intervento ha subìto tagli più consistenti ${ }^{21}$. Poiché il primo indicatore è estremamente sensibile alle strategie di informazione dei governi, in quanto mira ad identificare sia $\mathrm{i}$ vincitori che $\mathrm{i}$ perdenti, fornisce un criterio di valutazione degli interventi di ridimensionamento del welfare a mio parere preferibile.

3) Se si sceglie il secondo degli approcci appena descritti, si deve precisare come misurare la differenza tra prima e dopo. $\mathrm{Si}$ può fare ricorso ad uno dei metodi seguenti:

- un confronto del potere di acquisto delle prestazioni per casi-tipo di beneficiari (tale indicatore va espresso come differenza percentuale tra il livello delle prestazioni nel tempo t1 e nel tempo $\mathrm{t} 2$, dopo aver diviso l'ammontare assoluto per un deflattore);

- un confronto del tasso di sostituzione del reddito delle prestazioni per casi-tipo di beneficiari (tale indicatore va espresso come differenza percentuale tra il livello delle prestazioni nel tempo t1 e nel tempo t2, essendo le prestazioni espresse in proporzione al reddito);

- un confronto, nel tempo $\mathrm{t} 2$, tra il livello ipotetico delle prestazioni alle condizioni previste dalla legislazione precedente e l'ammontare attuale delle prestazioni alle condizioni introdotte dalla nuova legislazione.

21 Si rimanda alla nota 15 che affronta lo stesso problema con riferimento ai dati di bilancio. 
Questi tre indicatori possono variare in modo indipendente e possono dare luogo a interpretazioni sostantive tra loro in disaccordo. L'ipotetica riduzione di una prestazione (dovuta, ad esempio, a un cambiamento nelle procedure di indicizzazione) potrebbe infatti essere compatibile con un aumento del suo potere di acquisto. Allo stesso modo, aumenti del potere di acquisto possono coincidere con oscillazioni del tasso di sostituzione del reddito ${ }^{22}$. Nel caso in cui l'analisi comparata degli interventi restrittivi su programmi diversi non possa fare affidamento su tutti questi indicatori insieme, il ricercatore dovrebbe almeno cercare di applicare lo stesso procedimento di operazionalizzazione a tutti i programmi considerati.

4) Oltre a misurare i cambiamenti nel livello delle prestazioni, occorre cercare di descrivere nel modo più accurato possibile anche le modificazioni intervenute lungo le seguenti dimensioni:

- durata delle prestazioni;

- condizioni di ottenimento e di sospensione (descritte in termini qualitativi e/o trasformate in dati quantitativi);

- disposizioni finanziarie (fonte e livello di contribuzione);

- finalità o formato di copertura dei singoli schemi.

5) È necessario, infine, riportare tutti i dati nella forma più sistematica (tabelle) e riproducibile possibile (piuttosto che fare ricorso a giudizi vaghi come «sostanziale», «profondo», «(non) drammatico», «non banale», «estensivo», «meno di quanto ci si potrebbe aspettare», e così via).

Nonostante le considerazioni talvolta derisorie riguardanti l'approccio quantitativo nella ricerca sociale, che sembrano essere diventate una mania tra $\mathrm{i}$ ricercatori più giovani ${ }^{23}$, rimango

22 Qualora si selezionino i tassi di sostituzione del reddito per il confronto, è altrettanto importante specificare se sono definiti a livello istituzionale (è il caso dell'assicurazione di disoccupazione tedesca le cui prestazioni ammontano al $60 \%$ del reddito precedente netto) o se sono calcolati dai ricercatori (dividendo il livello assoluto delle prestazioni per il reddito medio, come è necessario fare nel caso inglese).

23 Si vedano, ad esempio, Pierson $(1995,473$ e 1996, 157) e Borchert $(1995,243$, 322 ), il quale ha seguito questo tipo di impostazione in Germania. Nonostante lo scetticismo rispetto all'approccio quantitativo, una delle prove più convincenti a favore dell'idea di Pierson secondo cui in presenza di governi neo-conservatori i programmi means-tested sono più resistenti di quelli universalistici è la constatazione (ripresa dalla ricerca dell'Urban Institute: Bawden e Palmer 1984, 185) che i primi tre bilanci della presidenza Reagan hanno portato ad una riduzione delle spese per lo schema Medicare pari al 6,8\%, contro tagli del 2,8\% allo schema Medicaid (Pierson 1994, 137). Non bisogna però dimenticare che questi dati si riferiscono alla spesa aggregata e non ai livelli delle prestazioni. 
convinto che, se si vogliono ottenere delle previsioni empiricamente fondate sulla logica dei processi di ridimensionamento, sia indispensabile (per quanto laborioso) sviluppare indicatori di cambiamento istituzionale riproducibili e di tipo quantitativo ${ }^{24}$.

Dopo aver illustrato come applicare almeno alcune delle regole di base fin qui menzionate, cercherò ora di misurare come i programmi selettivi per i poveri abbiano dovuto fronteggiare la «tempesta di tagli» in Germania e negli Stati Uniti. L'attenzione verterà sui programmi selettivi di entrambi i paesi. L'obiettivo è quello di riesaminare due delle ipotesi principali proposte da Pierson, cercando di rispondere alle seguenti domande: 1) fino a che punto i beneficiari di programmi selettivi possono essere effettivamente considerati i vincitori della fase di ridimensionamento del welfare? 2) Sono emerse specificità nazionali nello sviluppo dei programmi dei due paesi, nonostante il grado di inclusione della classe media possa essere considerato costante?

\section{I programmi contro la povertà in Germania e negli Stati Uniti}

Germania. Ogni cittadino che si trovi in stato di bisogno e sia privo dei mezzi di sostentamento, ha diritto all'assistenza all'interno dello schema di assistenza sociale nazionale introdotto nel 1961 (sulla base della preesistente legislazione del 1924 e del 1953). Lo schema è strutturato per permettere agli individui di soddisfare uno standard di bisogno stabilito in base ai modelli di consumo dei gruppi a basso reddito. I benefici colmano il divario tra i mezzi privati accertabili di una famiglia e lo standard di bisogno considerato come minimo socialmente accettabile (il cosiddetto Regelsatz). Se la prova dei mezzi non rivela la presenza di alcun reddito privato accertabile, lo schema si fa carico dell'intero costo di raggiungimento dello standard di bisogno, che varia a seconda delle dimensioni della famiglia e, fino ad un certo punto, anche in relazione all'età dei suoi membri. Standard di bisogno e standard di pagamento sono pertanto identici. In aggiunta allo standard di pagamento, che copre la differenza tra lo standard di bisogno e i mezzi privati, lo schema si fa anche carico del costo della casa e di situazioni di

24 Per un esempio di come codificare e quantificare le disposizioni legislative in un caso di assicurazione contro la disoccupazione, si veda Alber (1981). 
emergenza specifiche. I benefici o lo standard di bisogno non sono indicizzati in base all'inflazione, ma sono sottoposti ad un adeguamento annuale.

I principi in base ai quali calcolare lo standard di bisogno sono definiti da una legge nazionale, mentre lo standard di pagamento attuale è determinato dalle autorità dei Länder. Le variazioni regionali sono tuttavia molto contenute dal momento che lo standard di bisogno per le persone singole - che è identico allo standard di pagamento regionale se colui che ne fa richiesta non ha un reddito accertabile - oscillava nel 1994 tra i 496 (Mecklenburg-Vorpommern) e i 521 marchi (Baden-Württemberg, Brema, Assia). Oltre alle prestazioni in denaro, lo schema fornisce in particolari circostanze anche prestazioni in natura (per esempio, l'assistenza medica). Mentre lo schema è regolato da una legislazione nazionale, $\mathrm{i}$ finanziamenti derivano per la maggior parte dalle autorità locali e, in modo assai meno consistente, dai governi statali. Il contributo del governo federale è del tutto trascurabile, facendosi carico soltanto delle spese (contenute) di quei cittadini tedeschi in stato di bisogno che vivono all'estero. Nel 1993, 5 milioni di persone, equivalenti al $6 \%$ della popolazione, hanno ricevuto questo tipo di prestazione. La spesa di 48,9 miliardi di marchi corrisponde all'1,5\% del $\mathrm{Pi}^{25}$.

In sintesi, la politica tedesca contro la povertà è basata su di un principio semplice e coerente, ha un alto grado di standardizzazione nazionale e non prevede schemi categoriali per gruppi specifici. Gruppi particolari come i ciechi o gli handicappati hanno diritto a ricevere prestazioni rivalutate o prestazioni specifiche previste dallo stesso schema nazionale onnicomprensivo.

Stati Uniti. A differenza della situazione tedesca, le politiche americane di tutela dei poveri sono estremamente complicate. Esiste infatti una pletora di programmi, in parte sovrapposti, che interagiscono tra di loro (tab. 1). Il tentativo di fare un po' d'ordine concettuale all'interno di questa sconcertante varietà di schemi categoriali deve fare i conti innanzitutto con il concetto di soglia nazionale di povert $\hat{a}^{26}$. Tale indicatore era stato

25 Elaborazione dei dati da Statistisches Taschenbuch 1995, tabelle 8.16, 1.2, 2.1.

26 In realtà esistono due versioni di tale soglia: la soglia di povertà del Bureau of the Census e la soglia di povertà dell'Office of Management and Budget. A meno che non sia chiaramente indicato, di seguito si farà riferimento alla prima. 
TAB. 1. I principali programmi di sostegno al reddito per i poveri negli Stati Uniti

\begin{tabular}{|c|c|c|c|c|c|}
\hline $\begin{array}{l}\text { Programma (anno di } \\
\text { introduzione) }\end{array}$ & Criteri di eleggibilità & $\begin{array}{l}\text { Spese annuali (mi- } \\
\text { liardi di \$) }\end{array}$ & $\begin{array}{l}\text { Numero di benefi- } \\
\text { ciari }\end{array}$ & $\begin{array}{l}\text { Livelli di prestazione } \\
\text { (media mensile e } \\
\text { min/max) }\end{array}$ & $\begin{array}{l}\text { Procedure di finan- } \\
\text { ziamento }\end{array}$ \\
\hline Food Stamps (1964) & $\begin{array}{l}\text { Prova dei mezzi (beni inferiori ai } \\
2.000 \$ \text {; reddito netto inferiore } \\
\text { alla soglia di povertà; reddito lor- } \\
\text { do inferiore al } 130 \% \text { di tale so- } \\
\text { glia). Qualificazione automatica } \\
\text { per i beneficiari Adfc, Ssi, Ga. }\end{array}$ & $\begin{array}{l}\text { Nel 1993: } \\
\text { spesa totale: } 26,3 \\
\text { prestazioni: } 23,0\end{array}$ & $\begin{array}{l}\text { Nel 1992: } \\
\text { 7,4 milioni di fami- } \\
\text { glie } \\
\text { Nel 1993: } \\
27 \text { milioni di persone }\end{array}$ & $\begin{array}{l}\text { Nel 1993: } \\
\text { 68\$ per persona }\end{array}$ & $\begin{array}{l}\text { Finanziamento fede- } \\
\text { rale delle prestazioni; } \\
\text { ripartizione dei costi } \\
\text { amministrativi tra li- } \\
\text { vello federale e livel- } \\
\text { lo statale }\end{array}$ \\
\hline $\begin{array}{l}\text { Aid for Families with } \\
\text { Dependent Children } \\
\text { (Afdc) (1935) }\end{array}$ & $\begin{array}{l}\text { Prova del reddito e dei mezzi } \\
\text { (reddito lordo inferiore al } 185 \% \\
\text { della soglia di povertà; reddito } \\
\text { netto sotto lo standard statale di } \\
\text { risposta ai bisogni). }\end{array}$ & $\begin{array}{l}\text { Nel 1993: } \\
\text { spesa totale: } 25,2 \\
\text { prestazioni: } 22,3\end{array}$ & $\begin{array}{l}\text { Nel 1993: } \\
5 \text { milioni di famiglie; } \\
14,1 \text { milioni di per- } \\
\text { sone }\end{array}$ & $\begin{array}{l}\text { Nel 1992: } \\
\text { 383\$ (Missouri: } 121 \\
\text { - Alaska: } 743 \text { ) } \\
\text { 134\$ (Missouri: } 134 \\
\text { - Alaska: 383) }\end{array}$ & $\begin{array}{l}\text { Ripartizione del fi- } \\
\text { nanziamento tra go- } \\
\text { verno federale, stata- } \\
\text { le e locale } \\
\text { (circa } 60 \% \text { spetta al } \\
\text { governo federale) }\end{array}$ \\
\hline $\begin{array}{l}\text { Earned Income Tax } \\
\text { Credit (Eitc) (1975) }\end{array}$ & $\begin{array}{l}\text { Prova del reddito per famiglie } \\
\text { con componenti che lavorano (e, } \\
\text { fino al 1994, con figli). Limite di } \\
\text { reddito: } 23.050 \$ \text { (1993). }\end{array}$ & $\begin{array}{l}\text { Nel 1993: } \\
\text { spesa totale: } 10,9 \\
\text { (quota eccedente la } \\
\text { copertura fiscale pre- } \\
\text { vista dai saldi di bi- } \\
\text { lancio) }\end{array}$ & $\begin{array}{l}\text { Nel 1993: } \\
14 \text { milioni di fami- } \\
\text { glie }\end{array}$ & $\begin{array}{l}\text { Nel 1993: } \\
79 \$ \text { per famiglia }\end{array}$ & $\begin{array}{l}\text { Credito fiscale sul } \\
\text { reddito federale (dif- } \\
\text { ferenza tra totale del } \\
\text { credito e quota de- } \\
\text { stinata all'erario fe- } \\
\text { derale) }\end{array}$ \\
\hline $\begin{array}{l}\text { Low-Income Home } \\
\text { Energy Assistance } \\
\text { Program (Libeap) } \\
\text { (1981) }\end{array}$ & $\begin{array}{l}\text { Prova del reddito (la più severa } \\
\text { definizione di soglia di reddito } \\
\text { possibile: } 110 \% \text { della soglia di } \\
\text { povertà) e bisogno di assistenza } \\
\text { energetica. I criteri sono fissati } \\
\text { dagli Stati in modo discrezionale. }\end{array}$ & $\begin{array}{l}\text { Nel 1993: } \\
\text { spesa federale totale: } \\
1,3\end{array}$ & $\begin{array}{l}\text { Nel 1993: } \\
5,4 \text { milioni di fami- } \\
\text { glie (per l'assistenza } \\
\text { energetica) }\end{array}$ & $\begin{array}{l}\text { Nel 1993: } \\
\text { 15\$ (Louisiana e } \\
\text { South Carolina: } 7,5- \\
\text { Connecticut: 40) }\end{array}$ & $\begin{array}{l}\text { Block grants federali } \\
\text { ai singoli Stati }\end{array}$ \\
\hline
\end{tabular}


TAB. 1. (segue)

\begin{tabular}{|c|c|c|c|c|c|}
\hline $\begin{array}{l}\text { Programma (anno di } \\
\text { introduzione) }\end{array}$ & Criteri di eleggibilità & $\begin{array}{l}\text { Spese annuali (mi- } \\
\text { liardi di \$) }\end{array}$ & $\begin{array}{l}\text { Numero di benefi- } \\
\text { ciari }\end{array}$ & $\begin{array}{l}\text { Livelli di prestazione } \\
\text { (media mensile e } \\
\min / \max \text { ) }\end{array}$ & $\begin{array}{l}\text { Procedure di finan- } \\
\text { ziamento }\end{array}$ \\
\hline $\begin{array}{l}\text { Special Supplemental } \\
\text { Food Program for } \\
\text { Women, Infants and } \\
\text { Children (Wic) } \\
\text { (1966) }\end{array}$ & $\begin{array}{l}\text { Categoriale (donne incinte } \mathrm{e} \text { in } \\
\text { puerperio) con prova del reddito } \\
\text { (185\% della soglia di povertà) e } \\
\text { presenza di rischio nutrizionale. }\end{array}$ & $\begin{array}{l}\text { Nel 1993: } \\
\text { spesa federale: } 2,8\end{array}$ & $\begin{array}{l}\text { Nel 1993: } \\
5,9 \text { milioni di perso- } \\
\text { ne (donne, neonati, } \\
\text { bambini; il } 40 \% \text { di } \\
\text { tutti i bambini nati } \\
\text { in Usa) }\end{array}$ & $\begin{array}{l}\text { Nel 1993: } \\
\text { 30\$ (media nazionale } \\
\text { del costo del pacco } \\
\text { viveri mensile) }\end{array}$ & $\begin{array}{l}\text { Finanziamento fede- } \\
\text { rale; amministrazione } \\
\text { statale e locale }\end{array}$ \\
\hline $\begin{array}{l}\text { Supplemental } \\
\text { ty Income (Ssi) } \\
(1972)\end{array}$ & $\begin{array}{l}\text { Categoriale (sopra i } 65 \text { anni, cie- } \\
\text { chi o disabili) con prova del red- } \\
\text { dito (bisognosi con reddito infe- } \\
\text { riore a } 5.352 \$ \text { nel } 1994 \text { ). }\end{array}$ & $\begin{array}{l}\text { Nel 1993: } \\
\text { spesa totale: } 240 \\
\text { prestazioni federali: } \\
20,7\end{array}$ & $\begin{array}{l}\text { Nel 1993: } \\
6 \text { milioni di persone }\end{array}$ & $\begin{array}{l}\text { Nel 1993: } \\
\text { 317\$ (media federale } \\
\text { delle prestazioni SS) }\end{array}$ & $\begin{array}{l}\text { Finanziamento fede- } \\
\text { rale con integrazioni } \\
\text { statali }\end{array}$ \\
\hline $\begin{array}{l}\text { General Assistance } \\
\text { (Ga) (su iniziativa } \\
\text { dei governi statali e } \\
\text { locali) }\end{array}$ & $\begin{array}{l}\text { Persone bisognose al di sotto del- } \\
\text { la prova dei mezzi fissata dai sin- } \\
\text { goli Stati, le quali non accedono } \\
\text { ai programmi federali o necessita- } \\
\text { no di ulteriore assistenza. }\end{array}$ & & $\begin{array}{l}\text { Nel 1992: } \\
1,1 \text { milioni di perso- } \\
\text { ne } \\
\text { (in } 36 \text { Stati) }\end{array}$ & $\begin{array}{l}\text { Livelli più bassi e } \\
\text { durata più breve ri- } \\
\text { spetto alle prestazio- } \\
\text { ni federali }\end{array}$ & $\begin{array}{l}\text { Governi statali e lo- } \\
\text { cali }\end{array}$ \\
\hline
\end{tabular}


messo a punto in origine per ragioni statistiche. Si trattava della misura della quantità di denaro necessaria per acquistare una dieta nutrizionalmente adeguata al costo più basso possibile per una famiglia di una determinata dimensione: una famiglia con risorse inferiori a tre volte tale ammontare era considerata povera. Attualmente la soglia di povertà viene ridefinita ogni anno incrementando la soglia dell'anno precedente per il cambiamento dell'indice dei prezzi al consumo.

$\mathrm{Da}$ un prospettiva tedesca, l'aspetto distintivo delle politiche americane di tutela dei poveri è che trovarsi al di sotto di tale soglia ufficiale di povertà ha davvero poco a che fare con il diritto a ricevere le prestazioni di sostegno al reddito. Solamente il programma federale Food stamps (buoni viveri) mette più $o$ meno in diretta relazione il diritto alla prestazione con la definizione nazionale di povertà. Le famiglie il cui reddito accertabi$\mathrm{le}^{27}$ non supera la soglia di povertà definita a livello federale hanno infatti diritto ai buoni viveri. Il programma dei buoni viveri può essere considerato per questa ragione lo schema contro la povertà più inclusivo degli Stati Uniti, coprendo il 69\% della popolazione povera (che vive cioè al di sotto della linea di povertà descritta sopra) (Green Book 1994-95, 777). L'ammontare delle prestazioni che una famiglia riceve mensilmente è determinato in base alle dimensioni e al reddito della famiglia. Le famiglie senza reddito ricevono un ammontare uguale al $103 \%$ del costo mensile di una dieta nutrizionalmente adeguata (Thrifty Food Plan). Tale ammontare è aggiornato annualmente sulla base dell'aumento dei prezzi dei viveri. Nel 1993, la prestazione piena per una famiglia di quattro persone senza reddito ammontava a 375 dollari. Una famiglia con reddito riceve invece una prestazione che equivale alla differenza tra l'ammontare massimo e il $30 \%$ del reddito accertabile. Il governo federale finanzia l'intero costo delle prestazioni per $\mathrm{i}$ buoni viveri attraverso il gettito fiscale, mentre i costi amministrativi sono condivisi con le autorità statali.

Coloro che non superano la prova dei mezzi ed hanno figli di età inferiore ai 19 anni hanno diritto a ricevere delle prestazioni in denaro all'interno dello schema Aid for Families with Dependent Children (Afdc). Si tratta di una indennità selettiva per i figli a carico più che di uno schema generale di assistenza

27 Esiste un certo numero di deduzioni tra cui il $20 \%$ di qualsiasi reddito da lavoro. 
ai poveri. Nel 1992 lo hanno ricevuto 13,6 milioni di persone, pari al 37\% della popolazione povera. L'eleggibilità è determinata in base ad uno «standard di bisogno» che varia da Stato a Stato e che di solito è ben al di sotto della soglia nazionale di povertà. Per ottenere la prestazione Afdc, una famiglia deve superare due prove del reddito, una prova del reddito lordo e una prova del reddito (netto) accertabile. Famiglie il cui reddito lordo supera il $185 \%$ dello standard statale di bisogno non hanno diritto a ricevere la prestazione. Se il reddito accertabile è inferiore al $100 \%$ dello standard di bisogno, viene ufficialmente riconosciuto che la famiglia si trova in uno stato di bisogno.

Tuttavia, esistono numerose particolarità che contribuiscono a complicare le procedure. Innanzitutto, gli standard di bisogno definiti politicamente a livello dei singoli Stati non solo sono al di sotto della soglia statistica nazionale di povertà, ma variano anche in modo consistente da Stato a Stato ${ }^{28}$. Lo standard di bisogno per una famiglia di tre persone dello Stato mediano (pari a 554 dollari) corrispondeva nel 1993 solo al $57 \%$ della soglia nazionale di povertà ${ }^{29}$. Una seconda particolarità è che lo standard di bisogno è semplicemente una soglia teorica, di solito molto più alta dell'attuale standard di pagamento stata$\mathrm{l}^{30}$. Ed è solo in base al secondo parametro che si stabilisce l'ammontare delle prestazioni, dal momento che $\mathrm{i}$ benefici sono calcolati in base alla differenza tra il reddito accertabile del richiedente e lo standard di pagamento statale. In otto Stati, tuttavia, la massima prestazione erogabile è persino inferiore allo standard di pagamento ${ }^{31}$. Nello Stato mediano, la massima prestazione pagabile corrispondeva nel 1994 al $72 \%$ dello standard statale di bisogno, pari ad appena il $38 \%$ della soglia di povertà nazionale (per una famiglia mono-parentale di tre persone) ${ }^{32}$.

Si comprende pertanto perché le autorità pubbliche ameri-

28 L'ammontare delle prestazioni per una famiglia di tre persone variava, nel 1994, da un minimo di 338 dollari nel Delaware ad un massimo di 1.648 dollari nel New Hampshire (Green Book 1994-95, 371-373).

29 Elaborazione dei dati da Green Book (1994-95, 367, 373).

30 Solo in otto Stati lo standard di pagamento coincide con lo standard di bisogno.

31 In questi casi lo standard di pagamento corrisponde, con una sola eccezione (Wyoming), allo standard di bisogno.

32 I dati dell'edizione 1993 (p. 658) e dell'edizione 1994-95 (p. 367) del Green Book sono tra loro discordanti. Nell'edizione 1993 la prestazione massima nello Stato 
cane non si assumano la responsabilità di portare le persone povere al di sopra della linea ufficale di povertà. Lo scopo dello standard «platonico» di bisogno statale è di permettere ai beneficiari di colmare il divario tra le prestazioni Afdc ricevute e lo standard ufficiale senza essere penalizzati in termini di riduzione delle prestazioni. Ciò che potrebbe sembrare, in una prospettiva europea, una sorta di schizofrenia istituzionalizzata, è in realtà espressione dell'ossessione americana per la presenza, all'interno dei programmi sociali, di incentivi al lavoro (Green Book 1994-95, 329).

La maggior parte delle famiglie che riceve il sussidio Afdc (l'82\% del totale) riceve anche i buoni viveri. Mentre i buoni viveri non sono conteggiati per ottenere l'accesso alle prestazioni Afdc, il programma per i buoni viveri considera le prestazioni Afdc come reddito accertabile e di conseguenza riduce, in base ad esse, il valore dei buoni (Green Book 1994-95, 33 ).

A differenza dei buoni viveri, le prestazioni Afdc non sono indicizzate. Sia lo standard di bisogno che lo standard di pagamento sono solo adeguati periodicamente dagli Stati in base alle loro disponibilità fiscali. Tuttavia, il Family Support Act del 1988 stabilisce che ogni Stato debba valutare il livello del proprio standard di bisogno e di pagamento una volta ogni tre anni (U.S. Department of Health and Human Services 1993, 68). Il programma Afdc è finanziato congiuntamente dal Governo federale e da quelli statali e locali attraverso il gettito fiscale. Il finanziamento federale corrisponde ad un ammontare compreso tra il 50\% e l' $80 \%$ delle prestazioni Afdc in uno Stato (con variazioni inversamente proporzionali al reddito annuale pro capite di uno Stato: $55 \%$ in media) e copre il $50 \%$ delle spese amministrative (Green Book 1994-95, 324).

Oltre ai due principali programmi di assistenza alla popolazione povera, altri due schemi rivestono un'importanza di carattere generale, poiché definiscono l'eleggibilità in base al reddito

mediano (pari a 367 dollari) ammonta al $42 \%$ dello standard di bisogno (pari a 867 dollari) per il gennaio 1993 mentre, in base all'edizione 1994-95, corrisponde al $72 \%$ (prestazione massima di 366 dollari e standard mediano di bisogno di 507 dollari nel gennaio 1994). Le tabelle dalla 10 alla 13 dell'edizione 1994-95 definiscono lo standard di bisogno in uno Stato mediano pari a 554 dollari nel gennaio 1993 (p. 373) suggerendo così che i dati dell'edizione 1993 (che hanno ovviamente considerato l'Illinois come Stato mediano per entrambi gli indicatori) potessero essere sbagliati. La percentuale dello standard di bisogno riportata nel testo si riferisce al 1994 mentre la percentuale della soglia nazionale di povertà si riferisce al 1993. 
e non in termini categoriali. Il primo schema, creato nel 1975, ̀̀ l'Earned Income Tax Credit (Eitc) che tutela i poveri che lavorano. Originariamente questo schema federale aveva un'impostazione di tipo categoriale, ed infatti limitava la copertura a quelle famiglie con figli a carico in cui almeno un membro lavorava pur percependo un salario inferiore ad una certa soglia di reddito. Nel 1993, tuttavia, la copertura è stata estesa anche ai lavoratori a basso reddito senza figli a carico. Questo schema è stato progettato per offrire degli incentivi al lavoro a quelle famiglie che pur lavorando hanno un reddito al di sotto del livello di povertà. Eroga un credito fiscale calcolato come percentuale del reddito da lavoro e prevede un tetto in corrispondenza di un ammontare massimo. Il credito d'imposta cresce pertanto all'aumentare del reddito da lavoro, fino a raggiungere il suo ammontare massimo in corrispondenza di un certo livello di reddito ${ }^{33}$. Al di sopra di questo limite, fissato dallo Stato, il credito d'imposta rimane ad un livello massimo fissato fino a che non viene raggiunta un'altra soglia di reddito. Oltre questa soglia il suo ammontare si riduce di una percentuale che diminuisce gradualmente fino a raggiungere un punto di pareggio (break-even point) in cui il credito di imposta si annulla. Il programma Eitc fornisce così ad una famiglia la possibilità di ridurre il carico fiscale del reddito oppure, se il credito d'imposta supera il carico fiscale, di ricevere un sussidio che funzioni come imposta negativa sul reddito ${ }^{34}$.

Le famiglie a basso reddito hanno anche diritto alle prestazioni dello schema federale Low-Income Home Energy Assistance Program (Liheap). Questo schema è operativo dall'anno fiscale 1982 e fornisce agli Stati dei sussidi vincolati (block grants) per aiutare le famiglie che ne hanno diritto ad affrontare i costi di riscaldamento delle abitazioni. Nessuna famiglia il cui reddito sia inferiore al $110 \%$ della soglia di povertà può es-

33 Questo livello è chiamato «limite sul guadagno accreditabile» oppure «reddito minimo per un credito massimo».

34 Dopo che nel 1986 tutti i limiti di reddito del programma Eitc sono stati indicizzati in base all'inflazione, il limite sui redditi da lavoro accreditabile corrispondeva all'incirca alla soglia di povertà per gli individui soli (nel 1992 era uguale al 105\% di tale soglia). La soglia a partire dalla quale inizia la diminuzione progressiva si avvicina al livello della soglia di povertà per una famiglia di tre persone, ovvero a circa la metà del reddito medio di coloro che godono della Social security. Il punto di pareggio corrisponde grosso modo al livello medio di reddito. Dal 1994, le soglie variano in base al numero dei figli a carico: la soglia è due volte più alta per le famiglie con due figli rispetto alle famiglie senza figli. 
sere privata di tale prestazione. Il limite superiore per l'eleggibilità corrisponde al $150 \%$ della soglia del reddito di povertà o al $60 \%$ del reddito dello Stato mediano, indipendentemente dal suo ammontare. Entro questi margini, gli Stati godono di una considerevole libertà di manovra nel determinare $i$ criteri di eleggibilità e le forme di assistenza energetica da fornire. Si stima che nel 1993 5,4 milioni di famiglie abbiano ricevuto questa prestazione. La media delle prestazioni mensili per l'assistenza energetica varia ampiamente da Stato a Stato da un minimo di 7,5 dollari in Louisiana e South Carolina ad un massimo di 40 dollari nel Connecticut (Green Book 1994-95, 842843).

Le persone in stato di bisogno che non si qualificano per ricevere nessuna delle prestazioni federali o che hanno comunque bisogno di ulteriore assistenza, possono richiedere l'assistenza generale (General Assistance) fornita dai governi statali e locali. I criteri di eleggibilità e i livelli di pagamento variano da Stato a Stato ma le prestazioni di solito sono erogate all'ammontare più basso previsto e per una durata inferiore a quella prevista dallo schema di sostegno al reddito finanziato dallo Stato federale. Solitamente i beneficiari sono disoccupati che hanno cessato di ricevere il sussidio di disoccupazione o persone senza figli, non eleggibili, dal punto di vista categoriale, per l'Afdc. Con una dimostrazione esemplare dell'importanza attribuita agli incentivi lavorativi, circa un terzo degli Stati non garantisce l'assistenza generale a quelle famiglie che non hanno almeno un membro che lavora. L'assistenza è di solito finanziata congiuntamente dai governi statali e locali, ma quasi un quarto di tutti gli Stati fa gravare la responsabilità esclusivamente sui finanziamenti locali. Nel 1992 più di un milione di persone hanno ricevuto l'assistenza generale (Sspus 1993, 75).

Oltre a questi programmi generali di sostegno al reddito, esistono molti programmi categoriali per gruppi specifici. Il più importante schema categoriale è il Supplemental Security Income (Ssi) che eroga prestazioni in denaro agli anziani, ai ciechi e ai disabili con bassi redditi. Lo Special Supplemental Food Program for Women, Infants and Children (Wic) è invece un programma federale di assistenza nutrizionale e sanitaria progettato per assistere le donne prima e dopo il parto, i neonati e i bambini fino a 5 anni ritenuti dagli esperti sanitari a rischio nutrizionale. Coloro che hanno diritto a questo tipo di assistenza ricevono dei buoni per poter ottenere dei viveri supplementari ad alto 
contenuto nutrizionale. Più del $40 \%$ dei neonati nati negli Stati Uniti è coperto da questo schema (Sspus 1993, 73).

Infine, esiste una pletora di programmi che perseguono obiettivi specifici. Il più importante tra questi è lo schema $\mathrm{Me}$ dicaid che fornisce prestazioni sanitarie alle persone con mezzi insufficienti. Nel 1992, più di 35,6 milioni di persone erano coperte da questo schema e hanno ricevuto una prestazione annuale media di quasi 3.000 dollari ${ }^{35}$. Tra gli altri programmi con scopi speciali troviamo il Federal Housing and Rental Assistance Program, sussidi federali per le mense scolastiche, vari programmi di formazione lavoro e ancora numerosi schemi categoriali come le prestazioni per i veterani di guerra, o vari schemi per bambini con bassi redditi (per esempio, programmi di formazione Head Start o vari programmi di assistenza per l'infanzia).

Una tipica combinazione di prestazioni per una famiglia povera consiste in prestazione Afdc, buoni viveri e Liheap (Bawden e Palmer 1984, 193). Prestazioni Afdc e crediti Eitc coincidono solo in un numero ridotto di famiglie poiché l'eleggibilità per l'Afdc è molto al di sotto del limite di reddito per l'Eitc. Dal momento che la soglia di reddito per i buoni viveri e per la prestazione Eitc è molto simile e corrisponde pressapoco al livello nazionale di povertà, queste due prestazioni si sovrappongono più frequentemente (Green Book 1994-95, 335). Astraendo dalla sconcertante varietà di schemi, concentrerò l'attenzione sui programmi più generali, analizzando con maggior dettaglio quanto è accaduto ai buoni viveri, allo schema Afdc e allo schema Eitc durante la fase di ridimensionamento del welfare.

\section{Politica dei tagli e specificità nazionali}

Germania. Il programma tedesco di assistenza sociale è andato ampliandosi fino alla metà degli anni settanta. Con il rallentamento dello sviluppo economico avvenuto negli anni seguenti ha avuto inizio una fase di ridimensionamento, caratterizzata dapprima da una serie di non-decisioni, che hanno la-

$35 \mathrm{Nel} 1992$ i dati sul formato di copertura dello schema Medicaid variavano da 28,4 milioni in base alla Current Population Survey (Green Book 1994-95, 788) a oltre 31 milioni di beneficiari secondo l'Health Care Financing Administration (Green Book $1994-95,798)$ e a 35,6 milioni di «iscritti» in base al Sspus $(1993,53)$. 
sciato immodificati i principi, risalenti agli anni settanta, di definizione dello standard di bisogno, e poi da una serie di tagli. Il primo di questi tagli è avvenuto ancora sotto il governo di coalizione socialdemocratico-liberale. Nel 1981, infatti, furono ridotti gli incrementi delle prestazioni per alcuni gruppi specifici e fu posto un tetto all'aggiustamento delle prestazioni in base all'inflazione (si veda nella tab. 2 l'elenco dei cambiamenti legislativi). Il nuovo governo conservatore, salito al potere l'anno seguente, continuò la politica di ridimensionamento del welfare abbassando, con la legge finanziaria del 1982 e del 1983, i criteri di eleggibilità. Nel 1984, tuttavia, un importante simbolo di legittimazione della politica di austerità venne meno: la Corte Costituzionale dichiarò incostituzionale una tassa straordinaria, volta a promuovere gli investimenti, che era stata imposta sugli strati percettori di redditi elevati, e ordinò che coloro che l'avevano pagata venissero rimborsati. Il nuovo governo aveva appoggiato l'introduzione di questa tassa intendendo in questo modo dimostrare la «simmetria sociale» delle proprie misure di austerità. Quando questa legge venne respinta dalla Corte Costituzionale a causa di alcuni dettagli tecnici, il governo si astenne dall'imporre nuove misure sugli strati più elevati. Questo portò ad una mobilitazione di massa contro la politica di austerità, che da allora in poi poté essere in modo convincente osteggiata come una «lotta di classe dall'alto».

Reagendo a questa diffusa resistenza, il governo deliberò alcuni miglioramenti delle prestazioni sociali, tra cui, nel 1985, un aumento dei benefici di assistenza sociale che ristabiliva il livello delle prestazioni fissato negli anni settanta. Ulteriori tentativi di contenere i costi dello schema di assistenza ai poveri introdotto solo dopo l'unificazione tedesca misero i bilanci delle autorità pubbliche in una situazione di forte tensione. Dapprima la disoccupazione femminile di massa nei nuovi territori portò ad alcuni aumenti delle prestazioni per i genitori singoli e per le donne incinte; dal 1995 l'attenzione è stata rivolta ad una riforma finalizzata a contenere l'esplosione di costi dello schema di assistenza in una situazione di elevata disoccupazione. Tuttavia, le iniziative avviate dal governo intese a dar corpo, nel 1995 e nel 1996, ad un'estesa revisione del sistema di protezione sociale sono state finora bloccate dal Bundesrat, controllato dai partiti di opposizione.

Gli indicatori quantitativi mostrano che lo sviluppo dei livelli di prestazione è stato più o meno in linea con lo sviluppo 
TAB. 2. I principali cambiamenti nello schema tedesco di assistenza ai poveri

Anno Evoluzione normativa dello schema di assistenza

1974 Ultima legge della fase di espansione del welfare state. Estensione dei diritti individuali attraverso: (a) aumento dei limiti di reddito compatibili con l'accesso alle prestazioni; (b) esenzione dei redditi/beni dei genitori dalla prova dei mezzi di colui che fa richiesta delle prestazioni.

1981 Prime misure di austerità: aumento della prestazione standard limitato al $3 \%$ (anziché adeguato all'inflazione); riduzione dei livelli di prestazione per alcune categorie di beneficiari; riduzione dell'ammontare di beni compatibile con i criteri di eleggibilità.

1982 La legge finanziaria limita l'aumento della prestazione standard al $2 \%$ e posticipa l'adeguamento di 6 mesi (con un aumento annuale dell' $1 \%$ a fronte di un tasso di inflazione al $3 \%$ ).

1983 La legge finanziaria indebolisce i diritti individuali, favorendo una maggiore discrezionalità amministrativa; l'aumento dell'indice del costo della vita diventa la soglia massima anziché il limite minimo per gli adeguamenti annuali delle prestazioni; la copertura dei costi della casa è limitata ai costi «ragionevoli», legati al limite superiore dello schema di assistenza abitativa.

1985 Cambia la definizione di standard di bisogno e aumentano dell' $8 \%$ le prestazioni; introduzione di un incremento speciale per i genitori singoli con un figlio a carico e riduzione del limite di età da 65 a 60 anni.

1990 Decreto sulle regole di calcolo delle prestazioni standard: i nuovi criteri si basano sul modello di consumo dei gruppi a basso reddito piuttosto che sulla definizione del costo di uno standard minimo di vita; nuova variazione delle prestazioni per $\mathrm{i}$ bambini in base all'età.

1990 Misure speciali di riduzione dei livelli di prestazione nei territori della ex Ddr.

1992 Aumento degli incrementi di prestazione per i genitori singoli e per le donne incinte.

1993 Ridefinizione dello standard di bisogno con riferimento a un criterio unitario; introduzione del principio della «less eligibility» (chi è abile al lavoro deve essere incoraggiato a lavorare e non a vivere di sussidi) per le prestazioni a favore dei gruppi a basso reddito (incluso il reddito da trasferimenti); innalzamento a 65 anni del limite di età per ottenere incrementi speciali per persone anziane; tetto del 2\% (1993/94) agli adeguamenti annuali delle prestazioni e del 3\% per i due anni seguenti fino al giugno 1996; miglioramento delle possibilità per combattere l'abuso delle prestazioni.

dell'economia e con la situazione del reddito di altri gruppi sociali quali gli operai o i pensionati (fig. 1). Dal 1977 al 1984 la fase di tagli alle prestazioni ha conosciuto un declino nello standard dei benefici pari all' $8 \%$ in termini reali, ma questa è stata anche una fase in cui gli operai e i pensionati hanno accettato una riduzione del loro potere di acquisto. Dopo il 1984 i livelli reali di prestazione hanno continuato a crescere fino a che la politica di ridimensionamento del welfare del 1993 ha messo fine a questo processo. Nel lungo periodo le prestazioni massi- 


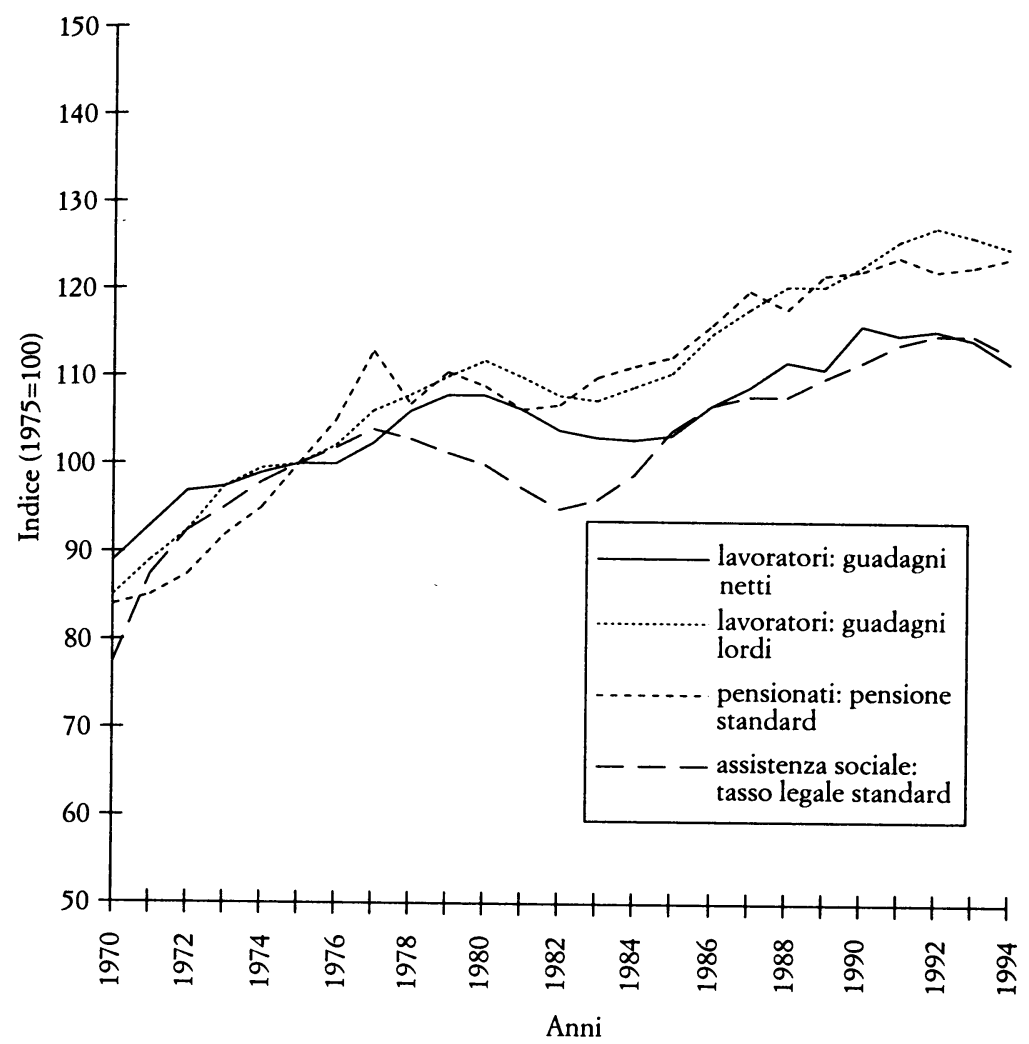

FIG. 1. Lo sviluppo dei livelli di prestazione e dei salari in Germania (ex-Germania ovest).

Fonti: Salari degli operai (totale degli stipendi netti/lordi e dei salari per persona occupata): Jahresgutachten des Sachverständigenrates zur Begutachtung der gesamt wirtschaftlinchen Entwicklung (1995/96 Bundestagsdrucksache 13/3016, 384). Pensioni standard: Statistiches Taschenbuch, Arbeits- und Sozialstatistik (1985, tab. 7.9 e 1995, tab. 7.9). Assistenza sociale: Bundesministerium für Arbeit und Sozialordnung (1994, Übersicht über das Sozialrecht, 626, 630). Deflattore: Bundesministerium fur Arbeit und Sozialordnung (1994), Materialband zum Sozialbudget (1993, 476); Statistisches Bundesamt, Statistisches Jabrbuch $(1995,636)$.

me di assistenza sociale si sono sviluppate all'incirca allo stesso tasso dei salari netti della forza lavoro dipendente. Il numero di beneficiari dello schema di assistenza sociale è raddoppiato tra il 1981 e il 1993 (aumentando da 2 a 4,3 milioni nella ex Re- 
pubblica Federale e a 5 milioni nell'intero paese), le pressioni finanziarie sono aumentate e hanno dato vita negli anni recenti ad un intenso dibattito su quale debba essere il livello adeguato delle prestazioni.

Lo sviluppo degli schemi di assistenza sociale è stato pertanto sottoposto a cicli di breve periodo che hanno seguito l'andamento economico e i deficit finanziari. Nel lungo periodo i beneficiari dello schema hanno più o meno mantenuto le loro posizioni rispetto alle altre categorie, per cui essi non sono usciti né vincitori né perdenti dalla fase di ridimensionamento.

Stati Uniti. I programmi americani contro la povertà sono stati oggetto di una serie di tagli sulla base della legislazione federale (si veda la tab. 3). I buoni viveri sono stati drasticamente tagliati nel 1980, 1981 e 1982: gli adeguamenti dei livelli di prestazione sono stati posticipati; come criterio di eleggibilità è stata introdotta una soglia di reddito lordo; alcuni gruppi particolari sono stati esclusi dal novero dei beneficiari; il livello delle prestazioni è stato abbassato. Un'inversione di tendenza nella politica di ridimensionamento si può osservare a partire dalla metà degli anni ottanta, dal momento che varie leggi - la più importante delle quali è stata l'Hunger Prevention Act nel 1988 - hanno revocato alcuni dei tagli e aumentato di nuovo le prestazioni.

I nostri indicatori quantitativi rivelano tuttavia che tali correzioni non hanno portato ai beneficiari miglioramenti in termini reali, ma sono riusciti a malapena a ristabilire lo status quo ante. Tra il 1980 e il 1988 il tasso grezzo dei beneficiari - che esprime il numero di beneficiari come percentuale della popolazione al di sotto della soglia di povertà - è diminuito del $7 \%$; negli anni novanta è tornato ai livelli della metà degli anni settanta (fig. 2) ) $^{36}$.

I dati sulle prestazioni mostrano quanto sia importante condurre operazionalizzazioni adeguate. A differenza delle prestazioni medie, che cambiano con il mutare della composizione dei beneficiari, la prestazione massima, erogata solo a coloro

36 I tassi dei beneficiari sono indicatori piuttosto grossolani dei processi di ridimensionamento del welfare. Occorre considerare tuttavia che essi sono influenzati non solo dalle trasformazioni istituzionali ma anche dai cambiamenti nella composizione della popolazione povera, e quindi tanto maggiore è il grado di povertà, tanto più elevate saranno le possibilità che più persone superino le prove di eleggibilità. 
TAB. 3. I principali cambiamenti nei programmi selettivi per $i$ poveri negli Stati Uniti

Food Stamps
1971 Livelli di prestazione uniformi.
1974 Estensione del programma: tutti gli Stati
sono tenuti a partecipare.

1977 La soglia di povertà diventa il nuovo limite di eleggibilità, per cui le famiglie devono superare delle prove dei mezzi (reddito/beni).

1980 Adeguamento annuale anziché semestrale dei livelli di prestazione; restrizione dei criteri di eleggibilità degli studenti.

1981 Obra: per la prima volta un criterio di eleggibilità basato sul reddito lordo viene applicato a tutte le famiglie senza un anziano o un disabile a carico; le deduzioni sul reddito vengono abbassate al $18 \%$; gli adeguamenti in base all'inflazione vengono posticipati; i lavoratori che partecipano agli scioperi vengono esclusi dal programma.

1982 Food Stamp Amendments: la prestazione massima è ridotta dal $100 \%$ al $99 \%$ del Thrifty Food Plan (Tfp); gli adeguamenti allo standard e le deduzioni di assistenza abitativa vengono posticipati all'ottobre 1983.

$1984 \mathrm{La}$ prestazione massima dei buoni viveri viene ripristinata al costo pieno del Tfp; viene introdotta una soglia di reddito netto per persone non anziane e non disabili in aggiunta alla soglia rispetto al reddito lordo; computo e adeguamento dei benefici vengono arrotondati per difetto (al dollaro inferiore).

Aid for Families with Dependent Children (Afdc)

1961 Introduzione dello schema Afdc-Up: le famiglie il cui capofamiglia è disoccupato hanno diritto ad una prestazione (nei primi 25 anni del programma, gli Stati potevano erogare prestazioni solo a famiglie mono-parentali con figli in stato di bisogno).

1967 Thirty and one-third rule: 30 dollari e un terzo del reddito aggiuntivo sono esentati dalla prova dei mezzi.

1971 La normativa federale stabilisce che un genitore per ottenere la prestazione Afdc debba lavorare meno di 100 ore in un mese per essere classificato come disoccupato (però alcuni Stati hanno incluso nello schema Afdc-Up famiglie in cui il principale percettore di reddito lavora anche 35 ore alla settimana).

1981 Obra: introduzione di una soglia di reddito che fa divieto agli Stati di erogare prestazioni alle famiglie con un reddito lordo superiore al $150 \%$ dello standard statale di risposta ai bisogni e richiesta agli Stati di considerare come reddito le prestazioni Eitc percepite mensilmente dai destinatari dell'Afdc che lavorano; la regola dell'esenzione dei 30 dollari e un terzo del reddito viene limitata a 4 mesi; vengono esclusi per 12 mesi dall'esenzione $\mathrm{i}$ beneficiari dell'Afdc che prima rinunciano alla copertura e poi ne fanno nuovamente richiesta; introduzione di un tetto alle deduzioni relative ai costi di assistenza per i figli e alle spese da lavoro.
Earned Income Tax Credit (Eitc)

1975 Tax Reduction Act: introduzione di crediti d'imposta pari al $10 \%$ dei primi 4.000 dollari; diminuzione graduale del $10 \%$ di reddito al di sopra di quel limite fino ad arrivare a zero in corrispondenza di un reddito lordo di circa 8.000 dollari.

1978 Revenue Act: il livello massimo del credito d'imposta è portato a 500 dollari (pari al $10 \%$ del reddito fino a 5.000 dollari); il livello massimo del credito d'imposta vale fino ad un reddito di 6.000 dollari; oltre tale soglia, diminuzione graduale del credito fiscale, fino allo zero in corrispondenza di un reddito di 10.000 dollari; è consentito anticipare il pagamento Eitc rispetto alla dichiarazione dei redditi annuale.

1979 Technical Corrections Act: sia la prestazione Eitc anticipata sia quella erogata sotto forma di somma complessiva devono essere considerate come reddito rispetto ai programmi Afdc e Ssi.

1981 Obra: la gestione del programma Afdc deve supporre che i destinatari dell'Afdc che lavorano ricevano il pagamento Eitc anticipato e deve pertanto ridurre l'ammontare della prestazione Afdc anche qualora il beneficiario non sia stato in grado di richiedere il pagamento Eitc anticipato.

1984 Deficit Reduction Act: il credito massimo è portato a 550 dollari (pari all'11\% del reddito fino a 5.000 dollari); la soglia di reddito è portata a 6.500 dollari; diminuzione graduale del credito 
TAB. 3. (segue)

Food Stamps

1985 Food Security Act: le famiglie in cui tutti i membri ricevono le prestazioni Afdc e Ssi sono rese eleggibili dal punto di vista categoriale per i buoni viveri; il reddito da lavoro e altre deduzioni vengono aumentate fino al maggio 1986; si richiede che tutti gli Stati attuino entro l'aprile 1987 un programma per l'occupazione e la formazione-lavoro per coloro che ricevono i buoni viveri.

1988Hunger Prevention Act: aumento della prestazione massima dei buoni viveri e specificazione degli aumenti futuri in percentuale al Tfp del giugno dell'anno precedente (1989: $100,65 \%$, 1990: 102, 05\%; 1991 e anni seguenti: $103 \%$ ) aumento della deduzione per l'assistenza ai familiari a carico da 160 dollari per famiglia a 160 dollari per persona dipendente; la prestazione Eitc non è più considerata reddito conteggiabile.

1992 Mancata diminuzione della prestazione per i buoni viveri, nonostante la leggera diminuzione del costo del Tfp; esenzione dei guadagni degli studenti fino all'età di 21 anni.

1993 Obra: aumento del tetto all'assistenza e della deduzione per l'assistenza ai figli o ad altri familiari a carico.
Aid for Families with Dependent Children (Afdc)

1984 Deficit Reduction Act: aumento della soglia di reddito lordo al $185 \%$ dello standard statale di risposta ai bisogni; l'esenzione dei 30 dollari viene portata a 12 mesi (mentre per un terzo del reddito il limite rimane a 4 mesi); la prestazione Eitc deve essere conteggiata solo quando è effettivamente percepita; viene ripristinato per 9 mesi lo schema Medicaid per coloro che ne hanno perso il diritto insieme con la perdita del diritto all'Afdc a causa del limite di 4 mesi per l'esenzione sul reddito. La normatva del 1981 è così in parte abrogata o attenuata.

1987 Obra: viene autorizzato un finanziamento federale pari al $75 \%$ per coprire i costi del programma statale di controllo degli abusi, ma viene vietata l'implementazione di norme che potrebbero limitare l'assistenza di emergenza per famiglie senza casa e per destinatari dell'Afdc particolarmente bisognosi.

1988 Family Support Act: si richiede agli Stati di esentare la prestazione Eitc nella determinazione dell'eleggibilità allo schema Afdc; cresce il livello di esenzione per le spese da lavoro e per i costi di assistenza ai figli; si richiede agli Stati di offrire entro l'ottobre 1990 programmi per l'occupazione e programmi di formazione (Jobs: Job Op)
Earned Income Tax Credit (Eitc)

fiscale, fino allo zero in corrispondenza di un reddito di 11.000 dollari; abrogazione delle norme del 1981: ai fini dell'erogazione della prestazione Afdc la prestazione Eitc deve essere considerata solo come reddito accertabile.

1986 Tax Reform Act: indicizzazione del credito e diminuzione graduale dei livelli in base all'inflazione; per il 1987 e il 1988 il livello di aumento viene specificato; innalzamento della soglia di entrata fiscale per le famiglie povere e indicizzazione della soglia in base all'inflazione.

1988Hunger Prevention Act: nel determinare i buoni viveri bisogna escludere la prestazione Eitc in quanto reddito accertabile.

1988 Family Support Act: nel determinare la prestazione Afdc bisogna escludere la prestazione Eitc in quanto reddito conteggiabile.

1990 Obra: il programma Eitc viene ampliato e vengono previste variazioni collegate alla dimensione della famiglia (le famiglie con due o più figli a carico hanno diritto a crediti di imposta più elevati); vengono introdotti due nuovi crediti supplementari (per i bambini al di sotto di un anno; per il premio di assicurazione sanitaria); aumentano i tassi del credito fiscale per una famiglia con un figlio a carico (dal $14 \%$ nel 1990 al $23 \%$ nel 1994); le prestazioni Eitc non devono essere considerate come reddito per determinare l'eleggibilità ai seguenti schemi: Afdc, Food stamps, Ssi, Liheap, Medicaid, assistenza abitativa per le persone a basso reddito. 


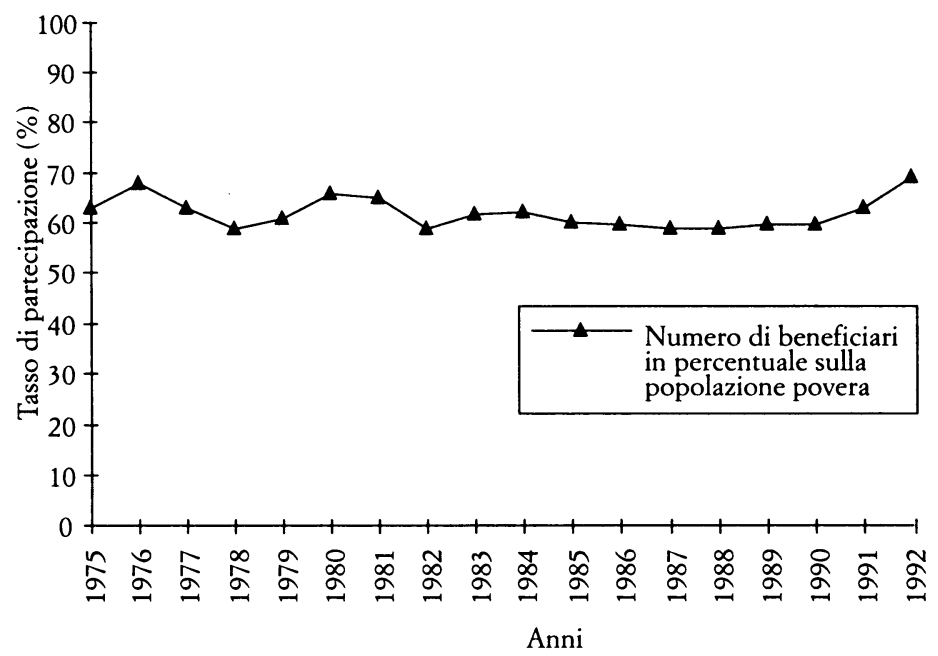

FIG. 2. Stati Uniti. I tassi di partecipazione al programma dei buoni viveri (numero di beneficiari in percentuale sulla popolazione povera).

Fonte: Elaborazione propria su dati da Green Book (1995, 777 e 1157).

che sono privi di reddito, può essere considerata un valido indicatore del cambiamento istituzionale. Durante gli anni ottanta solo le prestazioni medie sono aumentate in termini reali, superando persino i livelli del decennio precedente. Nello stesso periodo, $\mathrm{i}$ livelli delle prestazioni massime sono invece rimasti al di sotto dei livelli degli anni settanta e hanno oltrepassato di poco quei livelli negli anni novanta (fig. 3). Nel corso di venti anni, dunque, il potere di acquisto della prestazione massima per $\mathrm{i}$ buoni viveri è rimasto più o meno costante. I destinatari dei Food stamps, pertanto, non hanno beneficiato del progresso economico. Rispetto ai redditi, nel corso degli anni ottanta l'ammontare della prestazione massima è progressivamente diminuito. Per quanto negli anni novanta sia tornato a crescere, nel 1993 era tuttavia al di sotto di 3 punti percentuali rispetto al massimo storico degli anni settanta.

Dal momento che le prestazioni Afdc non sono indicizzate, esse subiscono effettivamente dei tagli in termini reali ogniqualvolta non sono adeguate in base ai cambiamenti dell'indice del costo della vita. A parte le riduzioni dovute a non-decisioni, prevalenti negli anni settanta, la principale politica di tagli è sta- 


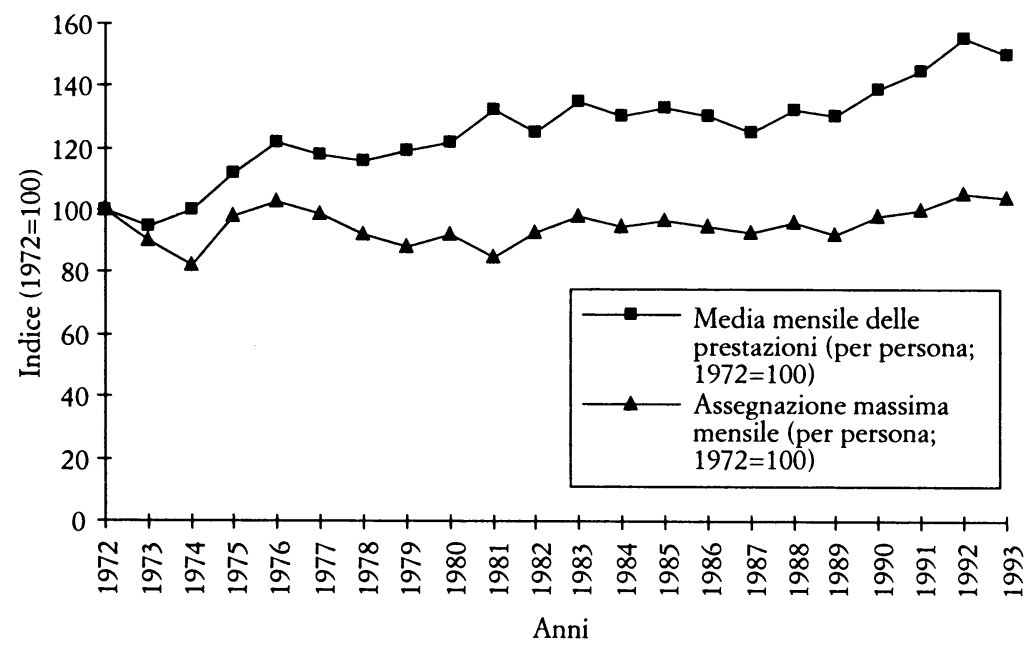

FIG. 3. Stati Uniti. Livelli di prestazione del programma buoni viveri (dollari costanti).

Fonti: Elaborazione propria su dati da Green Book (1995, 782); Economic Report of the President $(1996,343)$.

ta adottata nel 1981 quando l'Omnibus Budget Reconciliation Act (Obra) introdusse un limite di reddito lordo e abolì varie forme di esenzione del reddito. Da allora il credito Eitc conta come reddito anche se di fatto non viene percepito e l'esenzione di «30 dollari più un terzo del reddito da lavoro» è stata limitata a quattro mesi. Come conseguenza di questi cambiamenti 408.000 famiglie hanno perso il diritto a tale prestazione e 299.000 prestazioni sono state ridotte, con una perdita media in termini di prestazioni per famiglia di 1.555 dollari all'anno (equivalenti a 130 dollari al mese) (Green Book 1993, 738). Una parte di questi tagli è stata revocata dal Deficit Reduction Act del 1984 e dal Family Support Act del 1988 senza, tuttavia, che queste revisioni siano riuscite a riportare le condizioni dei beneficiari al periodo precedente l'anno 1981. Ed infatti il limite di reddito lordo come criterio di eleggibilità persiste tuttora (benché ad un livello più alto) e l'esenzione dei «30 dollari più un terzo del reddito da lavoro» rimane limitata a quattro mesi (Green Book 1994-95, 436).

Gli indicatori quantitativi confermano che i beneficiari dell'Afdc possono difficilmente essere considerati dei vincitori della fase di ridimensionamento. La percentuale della popolazione 


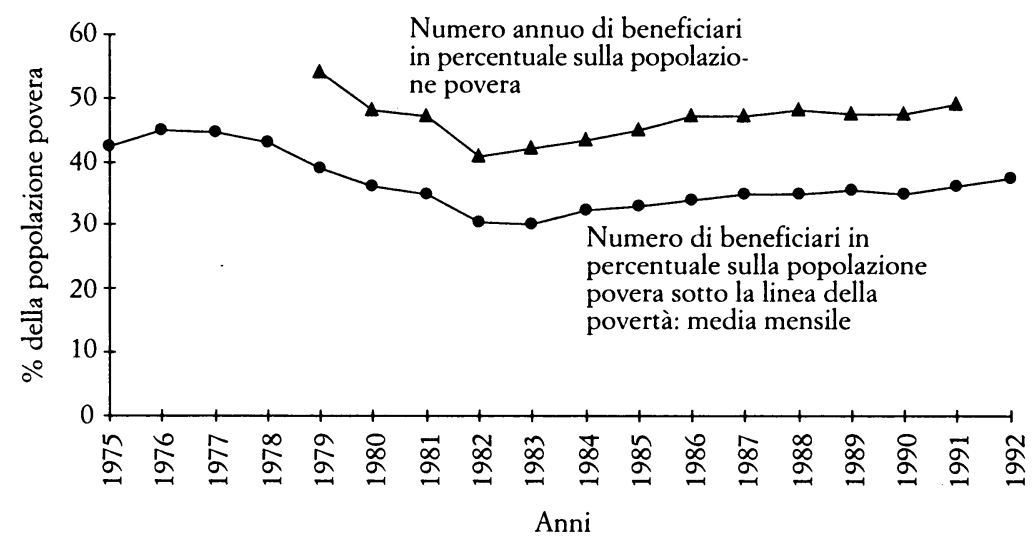

FIg. 4. Stati Uniti, prestazioni Afdc. Tassi di iscrizione (numero di beneficiari in percentuale sulla popolazione povera).

Fonti: Curva superiore: dati ufficiali da Green Book (1995, 399); curva inferiore: elaborazione propria su dati da Green Book (1995, 395 e 1157).

povera che riceveva le prestazioni è diminuita nel 1983 e negli anni seguenti non è più tornata ai livelli degli anni settanta (fig. 4) ${ }^{37}$. La soglia di povertà amministrativa all'interno dei singoli Stati è stata fissata persino a livelli più bassi. Nello Stato medio lo standard di bisogno di una famiglia di tre componenti è sceso dal $90 \%$ della soglia di povertà nazionale (1970) al $56 \%$ (1985). Pur aumentando leggermente in seguito, nel 1992 era ancora 31 punti percentuali al di sotto del livello del 1970. La prestazione mensile massima per una famiglia di quattro componenti è diminuita progressivamente, passando, nello Stato mediano, dal $67 \%$ della soglia nazionale di povertà (1970) al $36 \%$ (1992). In termini di potere d'acquisto, i livelli di prestazione sono caduti a picco durante gli anni settanta, poiché non venivano adeguati all'andamento dell'inflazione, ma hanno continuato a diminuire anche nel corso degli anni ottanta e novanta (fig. 5). Rispetto all'andamento del reddito, si registra una tendenza pressoché costante al declino, se si tiene conto che la

37 Di nuovo, questo indicatore ha una scarsa validità dal momento che non riflette solo i cambiamenti istituzionali. A parità di altri fattori, il tasso di iscrizione diminuirà se, per esempio, la proporzione di persone senza figli al di sotto della soglia di povertà aumenterà, in quanto solo i genitori hanno diritto a tale prestazione. 


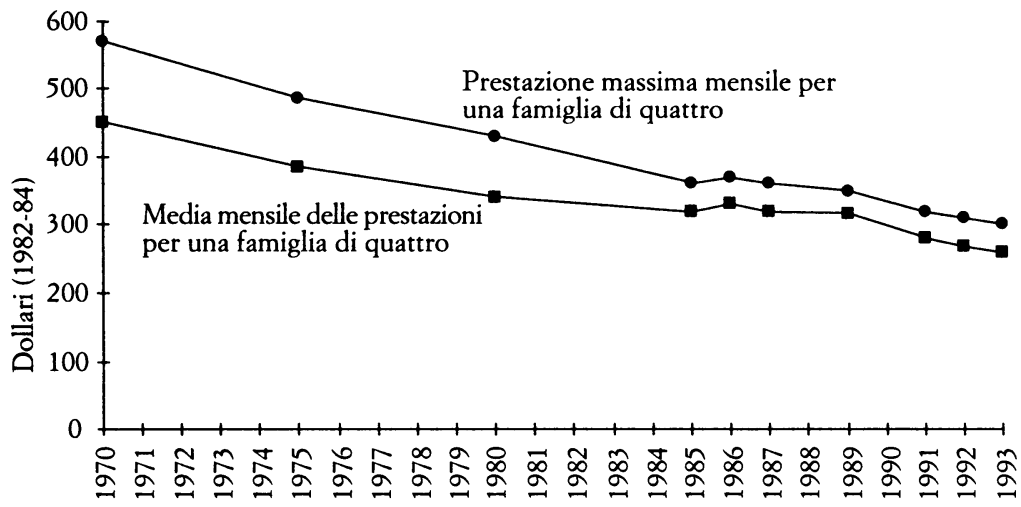

Anni

FIG. 5. Stati Uniti. Prestazioni Afdc in dollari costanti.

Fonti: Elaborazione propria su dati da Green Book (1995, 378); Economic Report of the President $(1996,343)$.

prestazione massima passa dal 46\% del reddito medio (1970) al $23 \%(1992)$.

Lo schema Eitc non ha subito alcun taglio esplicito. Tuttavia, come per il programma Afdc, una serie di non-decisioni sono state sufficienti a erodere i livelli di prestazione nel corso degli anni ottanta, dal momento che le soglie di reddito non sono state indicizzate. Alla metà degli anni ottanta il livello massimo del credito d'imposta era diminuito, in termini reali, di circa due terzi rispetto al suo livello originario e di due punti percentuali rispetto al reddito. Il Tax Reform Act del 1986 lo ha riportato al suo livello originario e ne ha più o meno stabilizzato il futuro potere di acquisto indicizzando i livelli rilevanti di reddito in base all'inflazione. L'Omnibus Budget Reconciliation Act del 1990 e del 1993 ha invece stabilito un aumento delle prestazioni, introducendo variazioni sulla base delle dimensioni della famiglia e un'estensione del diritto al credito di imposta per i contribuenti senza figli a carico.

Lo schema Eitc ha certamente esteso le prestazioni di sostegno al reddito ai poveri che lavorano, ma. la sua efficacia come incentivo al lavoro è stata in parte controbilanciata da un significativo abbassamento della soglia di entrata fiscale per le famiglie povere. Dal 1975 al 1984 la soglia fiscale è scesa infatti dal $122 \%$ all' $83 \%$ del livello di povertà, facendo così aumentare il 


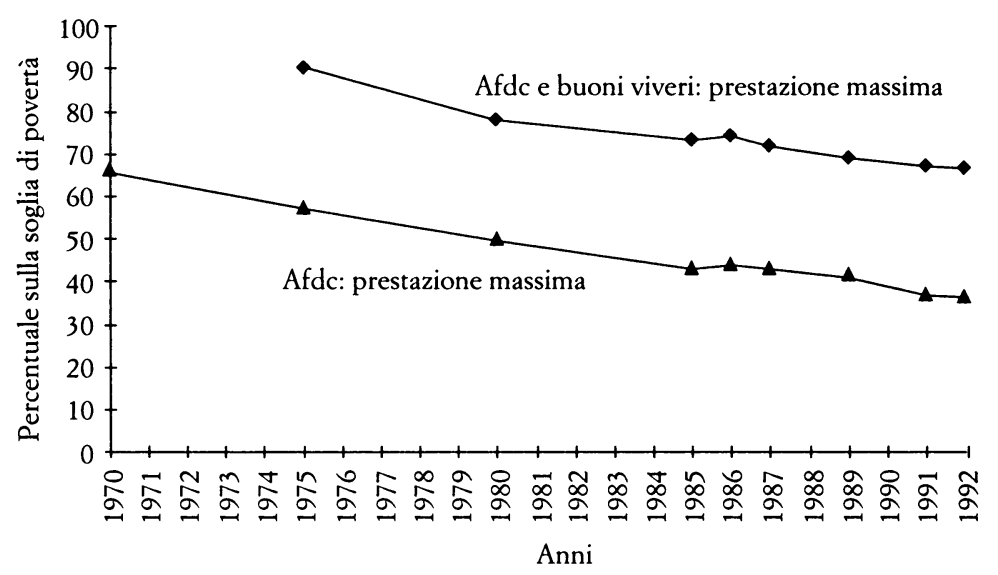

FIG. 6. Stati Uniti. Prestazioni Afdc e buoni viveri per una famiglia di quattro persone in percentuale sulla soglia nazionale di povertà.

Fonte: Elaborazione propria su dati da Green Book (1995, 378, 782, 1155).

carico fiscale delle famiglie povere. Quando il Tax Reform Act del 1986 ha aumentato il limite di reddito per il carico fiscale e lo ha indicizzato in base all'inflazione, la soglia è cresciuta di nuovo fino a corrispondere al $130 \%$ del livello di povertà (Green Book 1994-95, 730).

L'effetto aggregato che i cambiamenti legislativi hanno avuto sulla popolazione povera è difficile da quantificare. Per il nostro scopo anche un indicatore alquanto impreciso è sufficiente: si tratta di un indicatore che aggiunge la prestazione massima Afdc alla prestazione massima per $\mathrm{i}$ buoni viveri ${ }^{38}$. In base a questo indicatore la prestazione combinata per una famiglia di quattro persone è passata dal $90 \%$ della soglia nazionale di povertà nel 1975 al $67 \%$ nel 1992 (fig. 6). In altre parole, negli

38 Sebbene una combinazione tipica di prestazioni consiste delle erogazioni Afdc, dei buoni viveri e del Liheap - e per fasce di reddito più basse anche del beneficio Eitc - il nostro indicatore aggregato del sostegno al reddito non dovrebbe sottostimare il livello massimo di prestazioni raggiungibile. Le prestazioni massime, infatti, non sono erogate solo alle persone senza reddito (e quindi prive di Eitc) e i buoni viveri considerano la prestazione Afdc come reddito accertabile e si riducono di 0,3 dollari per ogni dollaro di reddito accertabile (Green Book 1993, 646). Le prestazioni massime di entrambi i programmi non possono pertanto coincidere; questa sovrastima probabilmente iper-compensa così l'esclusione del Liheap (per il quale non sono reperibili serie temporali di dati) dal pacchetto di prestazioni considerate. 
anni settanta un governo statale tipo (mediano) si assumeva la responsabilità di colmare quasi completamente il divario di povertà per una persona povera mentre negli anni novanta è in grado di colmare soltanto due terzi di tale divario. In termini reali, la prestazione combinata è diminuita del $27 \%$ nel lungo periodo. Data questa perdita di un quarto del potere di acquisto nel corso degli ultimi venti anni è difficile descrivere $i$ beneficiari dei programmi selettivi in America come dei vincitori della fase di ridimensionamento.

L'analisi comparata. I programmi tedesco e americano di assistenza ai poveri hanno in comune il fatto di essere entrambi altamente selettivi in quanto prevedono il superamento di stringenti prove dei mezzi. Entrambi, inoltre, non prevedono l'indicizzazione automatica della maggior parte delle prestazioni ma solo specifici adeguamenti annuali. Il primo aspetto implica l'esclusione delle classi medie, il secondo facilita i tagli attraverso non-decisioni politicamente poco costose. Un ulteriore elemento comune ai due paesi può essere individuato nel fatto che i primi attacchi sferrati dai governi neo-conservatori appena eletti (1981 negli Stati Uniti e 1983 in Germania) si sono esauriti negli anni ottanta e che alcuni dei primi tagli sono stati persino abrogati (nel 1984 negli Stati Uniti e nel 1985 in Germania). In Germania considerazioni di equità hanno esercitato un impatto sul processo di policy: infatti il governo si è mostrato debole di fronte alle critiche ogniqualvolta ha violato il principio della simmetria sociale in base al quale il peso delle politiche di ridimensionamento del welfare dovrebbe essere sostenuto in modo equo da tutte le fasce di reddito, sia quelle più alte che quelle più basse.

Il fatto distintivo, in termini comparati, è tuttavia la discrepanza di sviluppo di schemi selettivi simili nei due paesi. Mentre $\mathrm{i}$ beneficiari degli schemi americani di assistenza ai poveri hanno subito tagli consistenti che hanno spostato i livelli di prestazione sempre più lontano dalla soglia di povertà, le prestazioni di assistenza sociale in Germania sono aumentate dal punto di vista della dimensione in termini reali e hanno anche tenuto il passo con lo sviluppo di altre forme di reddito come le pensioni o i salari netti. Lo sviluppo dei programmi contro la povertà nei due paesi è stato sostanzialmente divergente. Questo si evince dalle figure 7 e 8 , che illustrano le traiettorie specifiche dei due paesi comparando lo sviluppo delle prestazioni in 


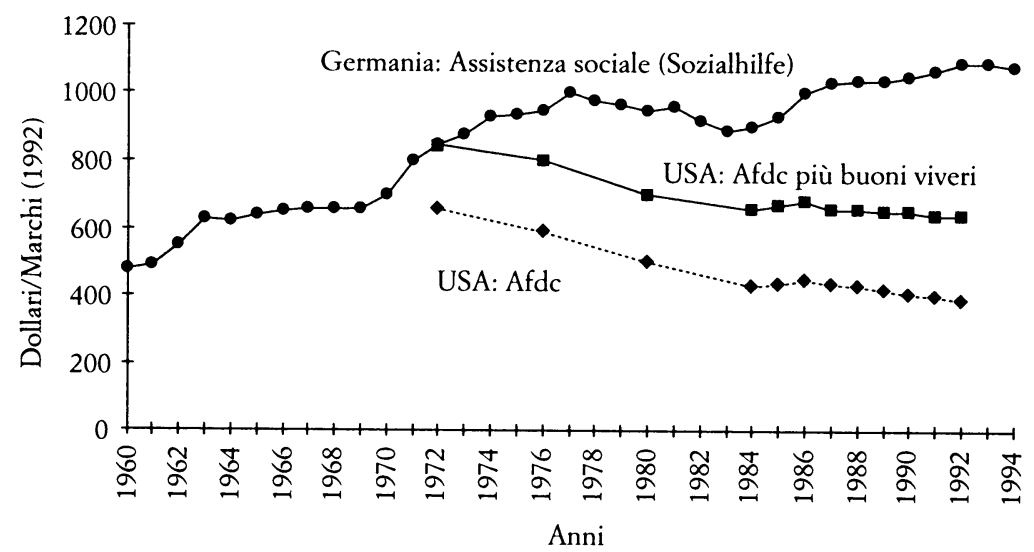

FIG. 7. Sviluppo delle prestazioni di assistenza sociale per una famiglia mono-parentale con due figli a carico negli Stati Uniti (dollari costanti, senza assistenza abitativa) e in Germania (marchi costanti).

Fonti: Elaborazione propria su dati da Green Book (1993, 1240); Übersicht über das Sozialrecht (1994, 626, 630); Statistisches Taschenbuch zür Arbeit- und Sozialstatistik (1950-1990, tab. 6.11 e 8.16); Materialband zum Sozialbudget (1993, 468, 476); Statistisches Jabrbuch $(1995,636)$.

termini reali e tracciando lo sviluppo delle prestazioni relative ai redditi da lavoro ${ }^{39}$.

La nostra analisi comparata dello sviluppo dei programmi selettivi per i poveri in Germania e negli Stati Uniti ci permette di trarre due conclusioni. La prima è che i beneficiari dei programmi selettivi non possono essere adeguatamente descritti come vincitori della fase di austerità. Negli Stati Uniti i destinatari delle prestazioni hanno sperimentato un peggioramento delle loro condizioni di povertà e si sono allontanati dalle condizioni di vita della popolazione attiva. In Germania lo standard di bisogno dei poveri è aumentato in termini reali così che lo standard delle loro condizioni di vita è migliorato nel tempo; tuttavia questo progresso ha significato solo tenere il passo con lo sviluppo dell'economia e con il progresso delle altre categorie. La seconda conclusione che si può trarre è che lo sviluppo

$39 \mathrm{Dal}$ momento che $\mathrm{i}$ tassi di sostituzione del reddito della figura 8 si basano su concetti di reddito da lavoro differenti e su definizioni diverse del diritto a ricevere piene prestazioni, il lettore si dovrebbe astenere dal confrontare i due livelli ed esaminare solo i diversi sviluppi nel tempo. 


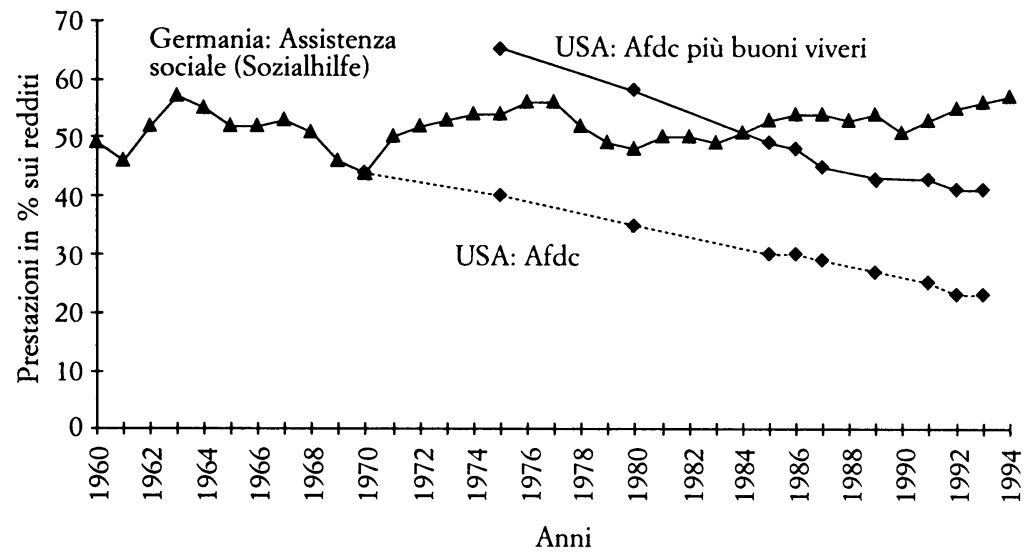

FIG. 8. Sviluppo delle prestazioni di assistenza sociale per una famiglia di quattro persone in percentuale sui redditi da lavoro medi negli Stati Uniti e in Germania (senza assistenza abitativa).

Fonti: Elaborazione propria su dati da Green Book (1993, 1240); Annual Statistical Supplement of the Social Security Bulletin (1995, tab. 4. B1); Übersicht über das Sozialrecht (1994, 626, 630); Statistisches Taschenbuch zur Arbeit-und Sozialstatistik (1950-1990, tab. 6.11 e 8.16); Materialband zum Sozialbudget 1993, 468, 476; Statistisches Jabrbuch $(1995,636)$. I redditi da lavoro sono definiti come somma degli stipendi e dei salari per una persona occupata per la Germania, e come media dei redditi da lavoro nella Social Security per gli Stati Uniti.

dei programmi selettivi è stato nettamente divergente nei due paesi nonostante il fatto che entrambi, prevedendo il superamento della prova dei mezzi, escludano le classi medie.

Mentre la prima di queste conclusioni contraddice l'attacco di Pierson alla dottrina corrente riguardante gli effetti dell'inclusione delle classi medie, la seconda lo sostiene. Dal momento che né i programmi tedeschi né quelli americani sono stati estesi alle classi medie, è ovvio che il grado di universalismo da solo non può spiegare le differenze di sviluppo e che le diverse traiettorie dei vari schemi dovrebbero essere collegate ad altri fattori come le caratteristiche di natura più specifica dei programmi.

\section{Alcune possibili ragioni dei diversi sviluppi di policy}

Perché lo sviluppo di lungo periodo dei programmi sociali mostra un'evoluzione così discrepante e in che modo questa diversa evoluzione può essere collegata alle caratteristiche dei programmi? Data la grande eterogeneità delle differenti regola- 
zioni statali, a questo punto posso avanzare delle ipotesi sul caso americano solo in termini generali. Innanzitutto e forse soprattutto, la popolazione povera americana non è tutelata da una Costituzione che obblighi lo Stato a garantire uno standard minimo di vita. In secondo luogo, l'assenza di uno schema nazionale e unificato di assistenza ai poveri ha molte conseguenze importanti. La frammentazione dei programmi sociali americani, sostenuti da strutture di interesse divergenti, tende ad impedire la formazione di potenti gruppi di interesse e contribuisce a definire la politica sociale come un interesse delle minoran$\mathrm{ze}^{40}$, mentre uno schema di assistenza ai poveri organizzato a livello nazionale fornisce un chiaro punto di riferimento per $\mathrm{i}$ gruppi di interesse facilitando così la loro mobilitazione. Dal momento che i programmi americani non sono solo frammentati da Stato a Stato ma anche a livello categoriale e prevedono molteplici regimi specifici per i vari gruppi a seconda delle situazioni particolari della vita, essi contribuiscono a forgiare lealtà di tipo particolare invece che un sostegno generale. Infine, l'organizzazione decentrata stimola probabilmente la competizione tra gli Stati e un dumping sociale che mira ad attrarre le imprese (attraverso oneri fiscali più bassi) e a prevenire l'eventuale migrazione dei poveri verso aree a normativa sociale più favorevole (attraverso severi requisiti di accesso alle prestazioni). Le opportunità di exit delle imprese ne risultano così ampliate, il potere di veto del mondo degli affari si accresce e gli interessi del capitale si intersecano con gli interessi dei contribuenti.

In sintesi, la situazione americana è caratterizzata da una pletora di programmi categoriali e decentrati che prevedono diversi criteri di eleggibilità per $i$ vari gruppi e una divisione dei poveri in «meritevoli» e «non-meritevoli». Per complicare ulteriormente questo quadro, la suddivisione della popolazione in varie categorie di beneficiari e la distinzione tra «chi lavora sodo e paga le tasse» e quei beneficiari che si suppone non meritino di ricevere le prestazioni di welfare sono intersecate dal tradizionale conflitto di classe tra il ceto medio e i poveri, così come dalle tensioni etniche tra bianchi e neri dal momento che

40 Questo dovrebbe anche risultare in un più dinamico ampliamento della differenza tra gli schemi pubblici e gli schemi della Social Security in America rispetto alla Germania. In questa sede un'analisi comparata dello sviluppo degli schemi pensionistici dei due paesi non può essere sviluppata. 
le prestazioni di welfare vanno in modo sproporzionato ai gruppi di minoranza. La divisione categoriale degli schemi rende pertanto difficile per i beneficiari unirsi nella difesa comune del welfare state, mentre l'intrecciarsi delle questioni legate allo stato sociale con i conflitti di classe e con quelli etnici aumenta il risentimento verso il welfare e persino il suo rifiuto (backlash) tra gli strati sociali più elevati (si vedano Edsall e Edsall 1991) ${ }^{41}$.

La notevole tenuta dello schema tedesco di assistenza ai poveri nel periodo di ridimensionamento del welfare è probabilmente collegata all'interazione di numerosi fattori. A parte l'impatto della garanzia costituzionale al diritto di assistenza, il carattere nazionale e non-categoriale dello schema di assistenza tedesco ha reso più semplice la creazione di una coalizione di associazioni di welfare, chiese e sindacati in difesa dello schema stesso. In secondo luogo - fattore probabilmente più importante - il governo federale che ha la competenza legislativa per gestire lo schema a livello nazionale non ha alcun interesse fiscale in gioco dal momento che lo schema è finanziato esclusivamente dai governi statali e locali. Poiché lo schema non minaccia le disponibilità finanziarie del governo federale, i decisori pubblici che operano a livello centrale non hanno sviluppato alcun interesse autonomo alla politica dei tagli.

Gli interessi fiscali del governo federale hanno cominciato ad essere chiamati in causa solo di recente, quando due sentenze della Corte Costituzionale (nel 1990) hanno dichiarato esenti dalle tasse tutti i redditi fino al livello di sussistenza, collegando la definizione di sussistenza minima al livello di assistenza sociale e obbligando il governo a varare una nuova legge fiscale entro il 1996. È da quel momento che il governo federale si è unito con l'associazione dei datori di lavoro in un attacco contro le prestazioni dello schema di assistenza ai poveri, accusate di essere troppo generose, di erodere gli incentivi lavorativi e di incoraggiare l'abuso delle prestazioni ${ }^{42}$.

41 In termini più generali il caso americano suggerisce anche che ci potrebbe essere un trade-off tra la cittadinanza politica e quella sociale nelle società ad alta immigrazione. Mentre queste società concedono abbastanza facilmente la cittadinanza politica, esse trovano più difficile, a causa della loro popolazione eterogenea, concedere la cittadinanza sociale che prevede diritti universali ed un minimo stabilito a livello nazionale.

$42 \mathrm{Nel} 1995$ il governo federale ha proposto una legge per la riforma dell'assistenza sociale che prevede le seguenti misure: contenimento dell'aumento annuale delle prestazioni in base alla crescita dei redditi da lavoro netti; riduzione del $25 \%$ delle prestazioni se colui che ne fa richiesta rifiuta un'offerta di lavoro adeguata; introduzione di 
Quello che i governi e le maggioranze parlamentari fanno quando sono al potere è presumibilmente in larga parte funzione di due fattori: ciò che i decision-makers vogliono e ciò che essi possono fare in presenza di limitazioni intrinseche ad un particolare assetto istituzionale e organizzativo. Rispetto agli orientamenti politici è importante sapere che la CDU (l'equivalente dei repubblicani americani) non è un partito neo-conservatore omogeneo. Si tratta di un partito cristiano con una forte componente operaia che fonda le proprie radici nel cattolicesimo sociale. Pertanto non solo gli interessi delle imprese ma anche quelli delle chiese e dei sindacati cristiani confluiscono all'interno di questo partito. Le chiese tedesche non sono solo degli osservatori esterni che valutano l'impatto delle politiche di welfare dal di fuori; al contrario i loro interessi sono incapsulati nelle politiche sociali in un duplice modo. Innanzitutto, le chiese hanno tradizionalmente una connessione con i partiti cristiani e in particolare con le correnti e gli organismi che rappresentano gli operai cristiani (Sozialausschuesse). In secondo luogo, esse controllano una larga parte delle associazioni di welfare, che funzionano come erogatori di servizi sociali per le persone bisognose che ricevono i sussidi. Ogni forma di ridimensionamento del welfare pubblico pertanto mette in pericolo la capacità delle associazioni di welfare di continuare ad erogare i servizi e di impiegare il personale. Le chiese tedesche perciò hanno chiari interessi in gioco nelle politiche sociali e mobilitano la resistenza all'interno della CDU quando vedono minacciato lo status quo. Data questa connessione con il cattolicesimo sociale organizzato, un cancelliere della CDU troverebbe difficile pensare attacchi al welfare state simili a quelli portati dal partito conservatore in Gran Bretagna o dai repubblicani negli Stati Uniti ${ }^{43}$.

integrazioni salariali per i datori di lavoro che assumono disoccupati di lungo periodo che ricevono le prestazioni sociali, e mantenimento delle prestazioni del $15 \%$ al di sotto del reddito netto delle categorie di reddito più basse della popolazione attiva. Il Bundesrat, tuttavia, ha rifiutato questa proposta di legge sostenendo che avrebbe gravato il governo locale di costi decisi dall'Ufficio Federale per l'Occupazione.

43 Dall'inizio degli anni novanta le chiese pubblicano rapporti sulla povertà e si sono appellate per combattere le nuove forme di povertà collegate all'emergere della disoccupazione conseguente all'unificazione tedesca. Prima delle più recenti elezioni federali le chiese cattoliche e protestanti hanno pubblicato una dichiarazione comune in cui chiedono al governo di occuparsi non solo della questione della competizione economica sui mercati internazionali, ma anche della qualità della vita dei bambini, degli anziani, dei disoccupati e dei poveri in Germania (Denkschrift «Zur wirtschaftlichen und sozialen Lage in Deutschland»). 
Rispetto alle strutture di opportunità politica c'è un importante vincolo sul governo federale dal momento che la legislazione sociale richiede il sostegno di entrambe le camere del Parlamento e il Bundesrat, a partire dall'unificazione tedesca, è sempre stato controllato dai socialdemocratici. Dal momento che il governo ha bisogno del sostegno dell'opposizione, importanti cambiamenti a livello di politiche sociali possono essere realizzati solo se sono stati attentamente preparati in circoli informali al di fuori dell'arena politico-elettorale.

Mentre la mancanza di controllo sul Bundesrat è un vincolo importante sul governo federale, le crescenti opportunità di exit per le imprese in un'era di globalizzazione rappresentano un secondo vincolo. Pressato dalle imprese e dalla banca federale, il governo ha recentemente optato per una nuova ondata di tagli allo schema di assistenza sociale e a vari altri programmi di welfare. Mentre dimostrazioni di massa non sono servite a fermare la riforma, non si è riusciti a superare il punto di veto ${ }^{44}$ del Bundesrat poiché i governi statali, compresi quelli guidati dalla CDU, hanno bocciato la riforma.

I recenti sviluppi tedeschi ancora una volta mostrano che $i$ costi politici dell'azione di governo sono multi-dimensionali, dal momento che i governi sono dilaniati da più parti in un conflitto di interessi tra legittimazione e accumulazione - $\mathrm{o}$, come hanno detto Marmor, Mashaw e Harvey (1990), in un conflitto tra «accessibilità» e «sostenibilità». Alla luce di questa tensione i policy-makers democratici sarebbero malconsigliati se prendessero in considerazione solo i costi politici dei tagli. Al contrario essi devono cercare di soddisfare sia gli elettori che gli interessi economici (cioè considerare le pressioni derivanti sia dal meccanismo di voice che di exit). Questo è tanto più vero se si considera che gli stessi elettori hanno due ruoli da giocare invece di uno solo, essendo essi tanto contribuenti quanto beneficiari dei programmi di welfare ${ }^{45}$.

Se i costi politici connessi all'una e all'altra dimensione tendono a neutralizzarsi a vicenda, il fatto che i governi siano vin-

44 Il concetto di «punti di veto» all'interno di un sistema politico è ripreso da $\mathrm{Im}$ mergut (1992).

45 Pertanto, sia gli elettori potenziali appartenenti al ceto medio che quelli appartenenti alla classe operaia sono favorevoli tanto ad una coalizione dal basso verso l'alto contro il big business quanto ad una coalizione dall'alto verso il basso contro il big government (cfr. Edsall e Edsall 1991). 
colati agli interessi degli elettori attraverso il processo elettorale non garantisce più che essi perseguano una politica che dia priorità alla soddisfazione dei bisogni dei beneficiari del welfare piuttosto che agli interessi di contenimento dei costi dei contribuenti, di coloro che pagano le tasse e degli investitori economici. Diventa allora probabile che il corso dell'azione sarà largamente dipendente dagli interessi fiscali immediati dello Stato stesso. In un contesto di deficit crescente, l'interesse immediato delle autorità di governo è quello di limitare le proprie responsabilità fiscali o tagliando quelle prestazioni che competono loro o scaricando la responsabilità di finanziamento su altri attori che non si trovano a controllare «punti di veto» durante il processo decisionale ${ }^{46}$.

Siccome il potere di prendere decisioni legittimamente vincolanti rimane nelle mani dello Stato - cioè dei partiti rappresentati nel governo tedesco e che controllano la maggioranza parlamentare - è probabile che gli interessi degli attori statali prevalgano sugli interessi degli attori sociali anche se questi ultimi possono essere ben organizzati in lobby. In ultima analisi, ciò che caratterizza lo Stato come attore è la sua prossimità all'arena del decision-making e la sua centralità in questa stessa arena piuttosto che il fatto che i suoi rappresentanti siano collegati agli interessi degli elettori attraverso il processo elettorale.

Queste considerazioni hanno delle importanti implicazioni di policy. Se si percepiscono le azioni dello Stato come rispondenti principalmente agli interessi degli elettori (e ai costi politici che si accumulano in questa dimensione), si è portati a ritenere che un ampio ricorso al gettito fiscale per il finanziamento del welfare state e una concentrazione di responsabilità a livello di governo centrale, caratterizzata da alta visibilità, costituiscano altrettante barriere contro la politica di ridimensionamento

46 La seconda cosa è successa ripetutamente in Germania in anni recenti, dove il governo federale è riuscito a spostare il carico finanziario del costo dell'unificazione tedesca dal bilancio federale sulle spalle dei contribuenti dello schema di assicurazione sociale. Pertanto, i contribuenti dell'assicurazione pensionistica (lavoratori, impiegati, datori di lavoro ma non i dipendenti pubblici e i lavoratori autonomi) hanno finanziato l'incremento che dovrebbe portare i pensionati della Germania dell'Est al di sopra della soglia di povertà mentre i contribuenti dell'assicurazione contro la disoccupazione hanno finanziato i costi delle politiche attive del mercato del lavoro per l'Est. La caratteristica distintiva è stato l'interesse del governo a limitare il peso del bilancio federale contro le resistenze unite del capitale e del lavoro (vale a dire i sindacati e le associazioni dei datori di lavoro in rappresentanza dei contribuenti degli schemi di assicurazione sociale che volevano mantenere $\mathrm{i}$ tassi di contribuzione ad un livello più basso). 
perché il governo è direttamente collegato agli elettori e non vuole essere ritenuto responsabile dei tagli. Se invece si percepisce il governo come un attore largamente autonomo con propri interessi fiscali e in grado di esercitare un controllo sull'arena del decision-making, allora il ricorso ai contributi sociali come fonte di finanziamento del welfare state e la diffusione di responsabilità fiscali appaiono come la forma di salvaguardia più efficace contro i tagli in un periodo di austerità che non un finanziamento statale centralizzato.

Il grado in cui i governi maturano un interesse alla politica di ridimensionamento di un particolare programma può essere messo in relazione con le caratteristiche del programma stesso e con il modo in cui quest'ultimo modella gli interessi fiscali del governo. Se il livello di governo che regola un programma di policy non contribuisce al suo finanziamento avrà poco interesse nel tagliarlo e lascerà lo schema relativamente intatto ${ }^{47}$. I tagli diventano probabili in quei campi di policy dove il livello di governo che ha la competenza legislativa per regolare quel campo ha anche la responsabilità fiscale in materia di finanziamento ${ }^{48}$.

A parte la struttura finanziaria, la dimensione di un programma è un altro fattore che determina gli interessi fiscali dei governi. Più piccolo è il numero dei beneficiari, più alto deve essere il sacrificio individuale per produrre un impatto notevole sul bilancio. Nel caso degli schemi con una copertura più ampia, invece, persino tagli modesti contribuiranno ad un risparmio aggregato. Tagli piccoli in grandi programmi pertanto appaiono come la soluzione migliore affinché un dato governo possa mediare tra gli interessi di controllo dei costi e gli interessi di soddisfazione dei bisogni. La misura in cui i governi perseguono tale via intermedia è probabilmente funzione delle alleanze che essi possono stringere con attori collettivi che rappresentano l'uno o l'altro questi interessi e i canali di accesso che collegano i gruppi di interesse all'arena del policy-making (per un riassunto schematico di questo punto si veda la fig. 9).

47 Questo è quanto è accaduto nello schema tedesco di assistenza ai poveri, ma potrebbe implicare il rischio di un rifiuto tra i livelli più bassi di governo contro gli obblighi privi di impegno di finanziamento da parte del governo federale come è successo negli Stati Uniti.

48 Questo è vero per la pensione tedesca e per l'assicurazione contro la disoccupazione con una condivisione del finanziamento e della legislazione federale. 


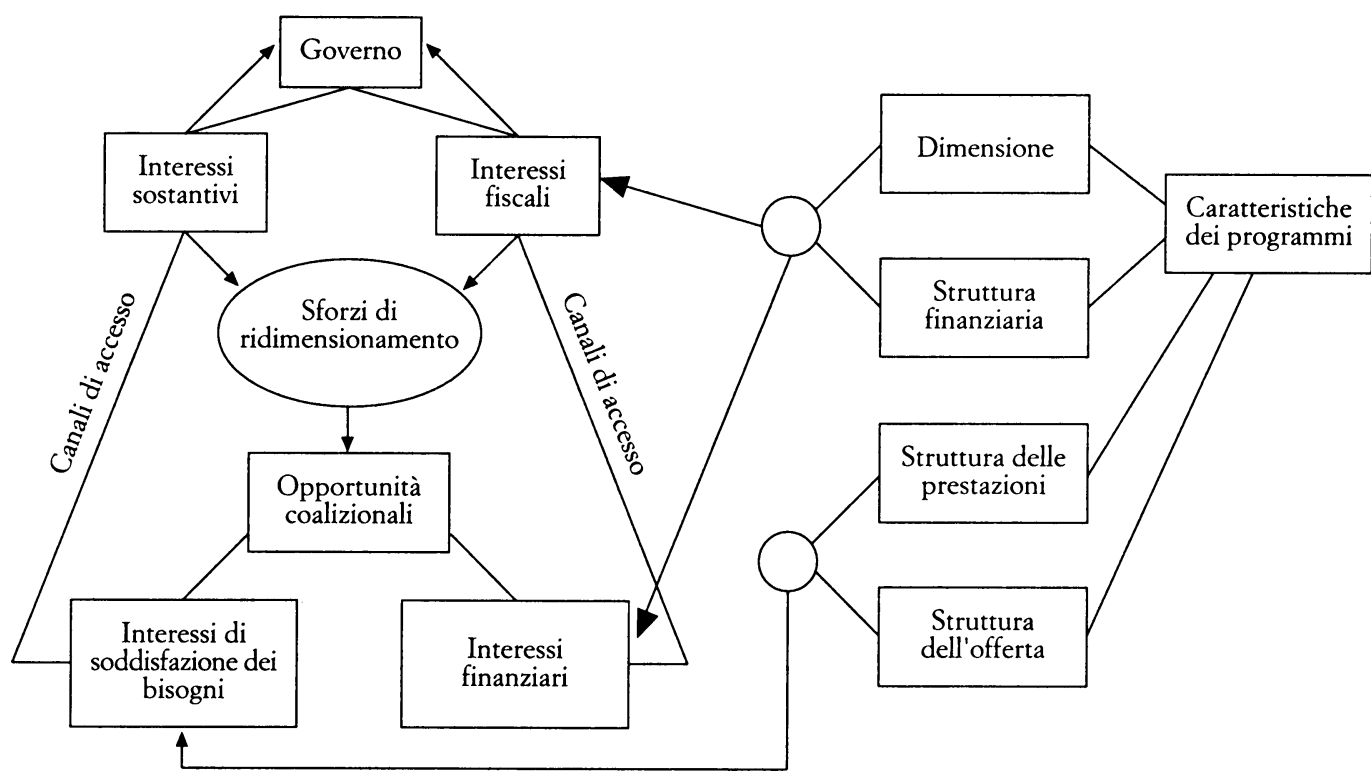

FIG. 9. Come concettualizzare le politiche di ridimensionamento del welfare. 


\section{Conclusioni}

Sul terreno descrittivo la nostra ricerca porta alla conclusione che ci sono marcate differenze specifiche fra paesi nello sviluppo dei programmi anche se viene mantenuto costante il grado di universalismo o di inclusione della classe media. Mentre non abbiamo ancora una conoscenza empirica sufficiente per giudicare se i programmi universali sopravvivono ai tentativi di ridimensionamento del welfare meglio dei programmi selettivi, è ormai chiaro che i beneficiari dei programmi selettivi non possono essere definiti vincitori della fase di ridimensionamento.

La prima conclusione conferma l'ipotesi di Pierson che la struttura dei programmi specifici è cruciale nel determinare il destino di uno schema. In aggiunta, comunque, per chiedersi chi è politicamente responsabile dei cambiamenti nei programmi bisogna anche chiedersi chi è responsabile dal punto di vista fiscale. Più alto è il deficit e più alta è la corresponsabilità finanziaria del livello di governo che ha il potere di legiferare su un programma particolare, più alte sono le probabilità che questo livello di governo maturi un interesse finanziario ai tagli. Che questo interesse finanziario dello Stato si trasformi in una politica restrittiva effettiva dipende dalle opportunità di coalizione del governo con attori collettivi che rappresentano gli interessi di coloro che si trovano a finanziare le prestazioni sociali da un lato e interessi di soddisfazione dei bisogni dall'altro. La soddisfazione dei bisogni dei gruppi marginali con poche risorse di potere ha la possibilità di affermarsi a seconda che gli altri attori sociali (le Corti, le chiese, i fornitori di servizi) funzionino come loro rappresentanti e possiedano dei canali di accesso al processo di policy-making ${ }^{49}$.

[traduzione di Franca Maino]

49 In aggiunta a questi gruppi di sostegno, i beneficiari di programmi selettivi potrebbero trovare dei partner coalizionali temporanei all'interno dell'apparato di governo, se gli interessi fiscali conducono i livelli inferiori di governo a richiedere nuove soluzioni di policy in grado di spostare il carico finanziario dal loro livello al livello centrale. Questo è avvenuto nello schema tedesco di assistenza di lungo periodo, in cui il peso del finanziamento è stato spostato da un programma selettivo di assistenza sociale ad un nuovo schema assicurativo universale finanziato con contributi accantonati, dal momento che i governi statali hanno fatto pressioni per essere sollevati da questo peso crescente sui loro programmi di welfare pubblici. 


\section{Riferimenti bibliografici}

Alber, J. (1981), Government Responses to the Challenge of Unemployment: The Development of Unemployment Insurance in Western Europe, in Flora e Heidenheimer, pp. 151-183.

- (1988), The West German Welfare State in Transition, in R. Morris (a cura di), Testing the Limits of Social Welfare. International Perspectives on Policy Changes in Nine Countries, Hanover-London, University Press of New England, pp. 96-134.

- (1992), Residential Care for the Elderly, in «Journal of Health Politics, Policy and Law», vol. 17, n. 4, pp. 929-957.

- (1995), A Framework for the Comparative Study of Social Services, in «Journal of European Social Policy», vol. 5, n. 2, pp. 131-149.

Atkinson, A.B. (1989), Poverty and Social Security, New York-London, Harvester Wheatsheaf.

Atkinson, A.B. e J. Micklewright (1989), Turning the Screw: Benefits for the Unemployed, 1979-1988, in Atkinson (1989), pp. 125-157.

Bawden, L. e J.L. Palmer, (1984), Social Policy: Challenging the Welfare State, in Palmer e Sawhill (1984), pp. 177-215.

Borchert, J. (1995), Die konservative Transformation des Woblfabrtsstaates. Großbritannien, Kanada, die Usa und Deutschland im Vergleich, Frankfurt-New York, Campus.

Borre, O.E. e E. Scarbrough (a cura di) (1995), Beliefs in Government. The Scope of Government, III Volume, Oxford-New York, Oxford University Press.

Bundesministerium für Arbeit und Sozialordnung (1995), Statistisches Taschenbuch 1995. Arbeits- und Sozialstatistik, Bonn.

Coughlin, R.M. (1980), Ideology, Public Opinion and Welfare Policy: Attitudes Toward Taxes and Spending in Industrialized Societies, Berkeley, Inst. of International Studies, University of California.

Dilnot, A. e A. Walker (a cura di) (1989), The Economics of Social Security, Oxford, Oxford University Press.

Edsall, T.B. e M.D. Edsall (1992), Chain Reaction. The Impact of Race, Rights, and Taxes on American Politics, New York-London, Norton.

Esping-Andersen, G. (1990), The Three Worlds of Welfare Capitalism, Cambridge, Polity Press.

Flora, P. (1986), Wachstum zu Grenzen - Stabilisierung durch Wandel. Zur bistorischen Lage der entwickelten Woblfabrtsstaaten Westeuropas, in M. Kaase (a cura di), Politische Wissenschaft und politische Ordnung. Analysen zu Theorie und Empirie demokratischer Regierungsweise, Opladen, Westdeutscher Verlag, pp. 27-39.

Flora, P. e A.J. Heidenheimer (a cura di) (1981), The Development of Welfare States in Europe and America, New Brunswick-London, Transaction Books (trad. it. Lo sviluppo del welfare state in Europa e in America, Bologna, il Mulino, 1983). 
Geißler, H. (1976), Die neue soziale Frage. Analyse und Dokumente, Freiburg, Herder.

Goodin, R.E. e J. Le Grand (a cura di) (1987), Not Only the Poor. The Middle Classes and the Welfare State, London, Allen \& Unwin.

Hanson, R.L. (1987), The Expansion and Contraction of the American Welfare State, in Goodin e Le Grand, pp. 169-199.

Heclo, H. (1974), Modern Social Politics in Britain and Sweden. From Relief to Income Maintenance, New Haven-London, Yale University Press.

Hirschman A.O. (1970), Exit, Voice and Loyalty. Responses to Declines in Firms, Organizations and States, Cambridge, Mass.-London, Harvard University Press (trad. it. Lealtà, defezione e protesta, Milano, Bompiani, 1982).

Immergut, E. (1992), Health Politics, New York, Cambridge University Press.

Klein, R. (1981), Values, Power and Policies, in Oecd, The Welfare State in Crisis, Paris, pp. 166-178.

Korpi, W. (1978), The Working Class in Welfare Capitalism. Work, Unions and Politics in Sweden, London-Henley-Boston, Rutledge $\&$ Kegan Paul.

Le Grand, J. e D. Winter (1987), The Middle Classes and the Defense of the British Welfare State, in Goodin e Le Grand, pp. 169-199.

Marmor, T.M., J.L. Mashaw e P.L. Harvey (1990), America's Misunderstood Welfare State. Persistet Myths, Enduring Realities, New York, Basic Books.

Morris, R. (a cura di) (1988), Testing the Limits of Social Welfare. International Perspectives on Policy Changes in Nine Countries, Hanover-London, University Press of New England.

O'Connor, J. (1973), The Fiscal Crisis of the State, New York, St. Martin's Press.

Offe, C. (1972), Politische Herrshaft und Klassenstrukturen. Zur Analyse spätkapitalisticher Gesellschaftssysteme, in G. Kress e D. Senghas (a cura di), Politikwissenschaft, Frankfurt, Europäische Verlangsanstalt, pp. 135-164.

- (1984), Contradictions of the Welfare State, London-Sidney, Hutchinson.

Orloff, A.S. (1993), The Politics of Pensions. A Comparative Analysis of Britain, Canada, and the United States, 1880-1940, MadisonLondon, The University of Wisconsin Press.

Orloff, A.S. e T. Skocpol (1984), Why Not Equal Protection? Explaining the Politics of Public Social Spending in Britain, 1900-1911, and the United States 1880s-1920, in «American Sociological Reviews, n. 49, pp. 726-750.

Palmer, J.L. e I.V. Sawhill (a cura di) (1984), The Reagan Record. An 
Assessment of America's Changing Domestic Priorities, Cambridge, Mass., Ballinger.

Pierson, P. (1994), Dismantling the Welfare State? Reagan, Thatcher, and the Politics of Retrenchment, Cambridge-New York, Cambridge University Press.

- (1995), Fragmented Welfare States: Federal Institutions and the Development of Social Policy, in «Governance», vol. 8, n. 4, pp. 449478.

- (1996), The New Politics of the Welfare State, in «World Politics», vol. 48, pp. 143-179.

Piven, F.F. e R.A. Cloward (1988), Why Americans Don't Vote, New York, Pantheon Books.

Roller, E. (1992), Einstellungen der Bürger zum Woblfabrtsstaat der Bundesrepublik Deutschland, Opladen, Westdeutscher Verlag.

Ruggles, P. e M. O'Higgins (1987), Retrenchment and the New Right: A Comparative Analysis of the Impacts of the Thatcher and Reagan Administration, in G. Esping-Andersen, M. Rein e L. Rainwater (a cura di), Stagnation and Renewal in Social Policy. The Rise and Fall of Policy Regimes, Armonk, N.Y., M.E. Sharpe, pp. 160-190.

Scholkopf, M. (1996), Altenpflegepolitik in Deutschland, University of Konstanz, mimeo.

Shapiro, R.Y. e J.T. Young (1989), Public Opinion and the Welfare State: The United States in Comparative Prespective, in «Political Science Quarterly», vol. 104, n. 1, pp. 59-89.

Svallfors, S. (1993), Policy Regimes and Attitudes to Inequality: A Comparison of Three European Nations, in T.P. Boje e S.E. Olsson Hort (a cura di), Scandinavia in a New Europe, Oslo, Scandinavian University Press, pp. 87-133.

Taylor-Gooby, P. (1985a), Public Opinion, Ideology and State Welfare, London-Boston, Routledge \& Kegan Paul.

- (1985b), The Politics of Welfare: Public Attitudes and Bebaviour, in R. Klein e M. O'Higgins (a cura di), The Future of Welfare, Oxford-New York, Basil Blackwell, pp. 72-91.

U.S. House of Representatives Committee on Ways and Means (1993), Overview of Entitlement Programs. 1993 Green Book Background Material and Data on Programs within the Jurisdiction of the Committee on Ways and Means, Washington, U.S. Government Printing Office.

- (1994), Where your Money Goes. The 1994-1995 Green Book, Washington-London, Brassey's.

Walker, A., J. Alber e A. Guillemard (1993), Older People in Europe: Social and Economic Policies. The 1993 Report of the European Observatory, Brussels, Commission of the European Communities. Wilensky, H. (1975), The Welfare State and Equality, Berkeley, University of California Press. 